MIT-CTP-2376

UFIFT-HEP-94-14

hep-th/9411047

\title{
THE DILATON THEOREM AND CLOSED STRING BACKGROUNDS
}

\author{
OREN BERGMAN ${ }^{\star}$ \\ Department of Physics \\ University of Florida \\ Gainesville, FL 32611, U.S.A. \\ BaRton ZwIEBACH $^{\dagger}$ \\ Center for Theoretical Physics, \\ LNS and Department of Physics \\ MIT, Cambridge, Massachusetts 02139, U.S.A.
}

\begin{abstract}
The zero-momentum ghost-dilaton is a non-primary BRST physical state present in every bosonic closed string background. It is given by the action of the BRST operator on another state $|\chi\rangle$, but remains nontrivial in the semirelative BRST cohomology. When local coordinates arise from metrics we show that dilaton and $|\chi\rangle$ insertions compute Riemannian curvature and geodesic curvature respectively. A proper definition of a CFT deformation induced by the dilaton requires surface integrals of the dilaton and line integrals of $|\chi\rangle$. Surprisingly, the ghost number anomaly makes this a trivial deformation. While dilatons cannot deform conformal theories, they actually deform conformal string backgrounds, showing in a simple context that a string background is not necessarily the same as a CFT. We generalize the earlier proof of quantum background independence of string theory to show that a dilaton shift amounts to a shift of the string coupling in the field-dependent part of the quantum string action. Thus the "dilaton theorem", familiar for on-shell string amplitudes, holds off-shell as a consequence of an exact symmetry of the string action.
\end{abstract}

$\star$ E-mail address: oren@phys.ufl.edu

Supported in part by D.O.E. contract DE-FG05-86ER-40272.

† E-mail address: zwiebach@irene.mit.edu, zwiebach@mitlns.mit.edu.

Supported in part by D.O.E. contract DE-AC02-76ER03069. 


\section{Introduction and Summary}

The soft dilaton theorem is a result relating an on-shell string amplitude with one zeromomentum dilaton and other physical states, to the on-shell string amplitude of the other physical states without the dilaton. The simple form that this relation takes leads to an interesting result. For the standard bosonic string background a shift in the vacuum expectation value of the dilaton field changes the dimensionless string coupling constant $\kappa$, and the slope parameter $\alpha^{\prime}$ (see [1]).

Following Yoneya [2], who first investigated the dilaton theorem in the context of lightcone string field theory, Hata and Nagoshi [3], Kugo and Zwiebach [4], and Hata [1], have studied this subject in the context of covariantized light-cone string field theories. Several conceptual issues related to operator ordering and the treatment of zero-momentum dilatons, which necessarily have zero string length, make it desirable to investigate the dilaton theorem in the context of covariant closed string field theory.

The dilaton theorem was studied in a more geometrical setting by Distler and Nelson [5], who concentrated on the ghost part $|D\rangle$ of the dilaton state. This BRST invariant physical state exists in every bosonic string background. Shifting the vacuum expectation value of the corresponding space-time field is expected to shift only the dimensionless coupling constant $\kappa$. The dilaton state $|D\rangle$ is not primary and is almost BRST trivial, namely $|D\rangle=-Q|\chi\rangle$, with

$|\chi\rangle$ a state that is illegal since it fails to be annihilated by $b_{0}^{-}[5]$. Distler and Nelson considered the correlator of $n$ physical states and a dilaton on a genus $g$ Riemann surface $\Sigma$, with the dilaton insertion integrated over the surface. The aim was to show that, schematically,

$$
\left\langle\int_{\Sigma} d^{2} z D(z, \bar{z}) \Phi_{1}\left(P_{1}\right) \cdots \Phi_{n}\left(P_{n}\right)\right\rangle_{\Sigma} \sim(2-2 g-n)\left\langle\Phi_{1}\left(P_{1}\right) \cdots \Phi_{n}\left(P_{n}\right)\right\rangle_{\Sigma} .
$$

This equation states that integrating a dilaton amounts to multiplication by the Euler number of the surface, thought of as having $n$ boundaries. This property, if generalized to the case when the $n$-insertions and the underlying moduli of the surface are also integrated, could be used to argue the dilaton theorem for on-shell string amplitudes. At first sight Eqn. (1.1) looks problematic because the dilaton, despite being physical, is not primary and therefore cannot be integrated on a Riemann surface unless one has fixed local coordinates at every point on the surface. The integral, a priori, depends on this choice. The main point of Ref.[5] is that while locally the integrand depends on the choice of coordinates, the integral could capture topological information that is independent of the choice of coordinates. This expectation is not fully realized. As emphasized by the authors, the ' $n$ ' contribution appearing in the above right hand side arises from "contact terms" and is ambiguous. The difficulty arises 
when the dilaton approaches any of the fixed punctures and as a consequence the surface approaches degeneration. In order to obtain a well-defined local coordinate for the dilaton one must parameterize the nearly degenerate surfaces. This can be done by factoring out a three punctured sphere. This three punctured sphere can be taken to be the standard twopunctured sphere, i.e. the $z$-sphere with local coordinates $z_{1}=z$ and $z_{2}=1 / z$, with an extra puncture at $z=1$. Upon a choice of a local coordinate at the extra puncture, one can calculate unambiguously the so-called contact term. It turns out that the contact term depends on the choice of this local coordinate, and therefore the left hand side of (1.1) is not well-defined. If the local coordinate is chosen to be symmetric under the exchange of the other two punctures the expected result arises $[5]$. $^{\ddagger}$ Our results corroborate that the above left hand side is indeed ambiguous, and requires a definition.

As the above discussion illustrates, the dilaton theorem is difficult to establish within first quantization (conformal field theory) due to the need to deal consistently with degenerate surfaces which must appear when considering string amplitudes. These difficulties do not arise in covariant closed string field theory, where the action does not involve nearly degenerate surfaces. Within string field theory the issue is to show that a shift of the string field dilaton changes the string field measure $d \mu e^{2 S / \hbar}$ in the same way as a change of $\kappa$ would. At the classical level this reduces to the statement that the change of the classical action upon a shift of the string field dilaton can be compensated by changing the string field coupling constant. The aim of the present paper is to prove conclusively the dilaton theorem using covariant closed string field theory. This problem is also a problem in background independence $[6,7]$, and raises new and subtle complications in the study of this issue. In this paper we will succeed in proving the closed string field theory dilaton theorem for the string field dependent part of the string field measure. The string field independent terms of the string field measure may or may not work out, we do not know at present. Such terms required careful treatment in the proof of background independence [7], and additional subtleties arise here.

One of the rather interesting surprises in the present analysis was finding a subtle but crucial difference between a conformal field theory (CFT) and a string background. While a CFT defines correlators on fixed Riemann surfaces, a string background includes forms on moduli spaces of Riemann surfaces. A string background is the proper data for the construction of a string field theory. We had expected that associated with the physical zero-momentum dilaton state there was a CFT deformation. While the non-primary nature of the dilaton creates complications, it is possible to write a deformation associated with the dilaton. The deformation, however, turns out to be trivial. The CFT is not deformed at all. More concretely, the

\footnotetext{
$\ddagger$ Distler and Nelson argued that the choice of a symmetric three punctured sphere was natural from the viewpoint of string field theory, and suggested further investigation of this issue.

$\S$ The Feynman rules construct the nearly degenerate surfaces in a way that is consistent with factorization.
} 
deformed correlators can be made to agree with the original correlators by a redefinition of the local operators of the theory. The redefinition is generated by the ghost number operator, and precisely reproduces the deformation induced by the dilaton by virtue of the ghost number anomaly. This means that the zero-momentum dilaton is not really a parameter of the conformal theory." Nevertheless the dilaton turns out to deform a string background. While we do not give an axiomatic definition of a string background we sketch a possible definition in sect. 7.2. To show that two apparently different string backgrounds are identical we must show that that all the forms can be made to coincide by a redefinition of the local operators of the conformal theory. Since forms on moduli spaces involve antighost operators, the similarity transformation induced by the ghost number operator, that cancels out the deformation for the conformal theory, does not cancel out the deformation of the string background. Thus the dilaton provides an example where the space of vacua of string theory has a parameter that is absent in the space of conformal field theories. This implies a subtle difficulty in attempting to use two-dimensional theory space as the precise arena for the formulation of a manifestly background-independent string theory. Further investigation of the difference between a CFT and a string background is likely to be fruitful. A string background that arises from a CFT will be called a conformal string background. It is certainly conceivable that there are string backgrounds that do not arise from CFT's.

At any rate, since string backgrounds related by a dilaton deformation are different, we can ask whether two string field theories constructed using two such backgrounds are the same. Proving they are the same amounts to showing that these backgrounds are backgrounds of the same string field theory. In this light it is clear that the dilaton theorem is a case study for background independence. It is a novel case because the different backgrounds originate from the same conformal theory.

In the analysis of background independence in Ref.[ 7], the fields to be shifted were BRST physical, primary fields of the form $c \bar{c} \mathcal{O}$, with $\mathcal{O}$ a primary, dimension $(1,1)$ field constructed out of the matter sector of the CFT. Since the dilaton is not primary, in order to insert it in some region of a Riemann surface we need a family of local coordinates, namely a local coordinate for every point in the region. Such families of local coordinates can be obtained by introducing metrics on the surfaces. To extract local coordinates from metrics we use a prescription given by Polchinski [ 8]. Such local coordinates are known in the mathematical literature as the $\omega$ normal coordinates associated to a Kähler metric with Kähler form $\omega$ (see Ref.[ 9], sect. 3.7). Here we will simply call them local normal-coordinates. Choosing the metrics that must be used to get the local normal-coordinates is a delicate matter. For this we must extend some

I The effect is analogous to the one where the $\theta$ parameter of QCD becomes unobservable in the presence of massless fermions. 
of the results of Ref.[7] concerning the $\mathcal{K}$ operator and the $\mathcal{B}$ spaces. The $\mathcal{K}$ operator acting on a surface $\Sigma$, equipped with coordinate disks $D_{i}$, gives the two dimensional space of surfaces represented by the surface $\Sigma$ with a new puncture inserted anywhere on $\Sigma-\cup D_{i}$. Acting on a space of surfaces, $\mathcal{K}$ will do this to each surface in the space. The local coordinate at the new puncture is not specified. $\mathcal{B}$-spaces, the new geometrical ingredient appearing in background independence, arise roughly as homotopies induced by $\mathcal{K}\left(\mathcal{B}_{g, n}\right.$ provides a homotopy between $\mathcal{K} \mathcal{V}_{g, n-1}$ and $\left.\mathcal{V}_{g, n}\right)$. Since $\mathcal{B}$ spaces always have a special puncture, and the local coordinate at this puncture is not defined, the recursion relations satisfied by the $\mathcal{B}$ spaces are weak equalities, namely, they hold up to the local coordinate at the special puncture. We define an operator $\overline{\mathcal{K}}$ that adds a puncture and specifies the local coordinate at the puncture. Acting on a surface, the operator $\overline{\mathcal{K}}$ uses the minimal area metric on that surface to extract the local normal-coordinates. We then show that the recursion relations for the $\mathcal{B}$ spaces can be turned into strong equalities, needed for dealing with dilatons. While in Ref.[ 7] $\mathcal{B}$-spaces had to have at least two punctures, one special and one ordinary, we find it useful to introduce higher genus $\mathcal{B}$ spaces with one special puncture and no ordinary puncture.

We use the results of Ref.[10] to compute the two-form associated with a $|D\rangle$ surfaceinsertion that uses the most general family of local coordinates. Similarly, we compute the one-form associated with a $|\chi\rangle$ line-insertion. Particular cases of this result were obtained in Refs.[ $8,5,11]$. When the family of local coordinates is the family of local normal-coordinates associated with a metric on the surface, the dilaton two-form reduces to the curvature twoform of the metric, in agreement with a result obtained earlier in Ref.[8]. Under the same circumstances we show that a line integral of the $|\chi\rangle$ one-form over a curve precisely computes the integral of the geodesic curvature over the curve. Since both the curvature two-form, and the geodesic curvature are elements of the Gauss-Bonnet theorem expressing the Euler number of a surface in terms of the Riemannian data on the surface, we see that both the $|D\rangle$ two-form and the $|\chi\rangle$ one-form must be present in a regularized version of (1.1). For a surface $\Sigma$ of genus $g$ with $n$ punctures, and equipped with coordinate disks $D_{i}$, the regulated form of Eqn. (1.1) takes the form, schematically,

$$
\left\langle\left(\int_{\Sigma-\cup D_{i}} d^{2} z D(z, \bar{z})+\int \underset{\partial\left(\Sigma-\cup D_{i}\right)}{\int} d \xi \chi(\xi)\right) \Phi_{1}\left(P_{1}\right) \cdots \Phi_{n}\left(P_{n}\right)\right\rangle_{\Sigma} \sim(2-2 g-n)\left\langle\Phi_{1}\left(P_{1}\right) \cdots \Phi_{n}\left(P_{n}\right)\right\rangle_{\Sigma} .
$$

Here the dilaton is only integrated over the surface minus the unit disks defining the coordinates at the punctures, and the $\chi$ operator is integrated over the boundaries of the unit disks. The expression is perfectly regulated since operators never collide. Unlike (1.1), the states inserted at the punctures need not be physical; this is essential in order to go off-shell. The insertions 
of $D$ and $\chi$ are done with the local normal-coordinates arising from an arbitrary metric on $\Sigma^{\star}$. We use the $D$ and $\chi$ forms to construct the CFT deformation related to (1.2), and to develop its generalization to the cases when we integrate over the positions of the external states and over the moduli of the surface.

The paper is organized as follows. Section 2 begins with a review of the basic properties of the dilaton state and of the procedure for extracting families of local normal-coordinates from metrics. We then review the BV (Batalin-Vilkovisky) algebra of Riemann surfaces and the corresponding BV algebra of string functionals. In section 3 we discuss modified versions of the $\mathcal{K}$ operator that insert a puncture on Riemann surfaces and define a local coordinate for the insertion. We also introduce an operator $\mathcal{L}$ that, acting on a surface, inserts a puncture on the coordinate curves. We review the properties of the homotopy spaces $\mathcal{B}$, and show how to obtain strict recursion relations taking into account the local coordinates at the inserted punctures. In section 4 we first motivate and then give a precise formulation of the dilaton theorem, indicating what needs to be proven. In section 5 we begin by reviewing the GaussBonnet theorem in two dimensions, paying particular attention to the contribution of geodesic curvature. We then compute the dilaton two-form and the $|\chi\rangle$ one-form for the case of a general family of local coordinates. When the local coordinates arise from a metric, the dilaton twoform and $|\chi\rangle$ one-form are shown to become the curvature two-form and the geodesic curvature respectively. In section 6 we derive two general results concerning the insertion of the dilaton and of the state $|\chi\rangle$ in spaces of Riemann surfaces. The insertions are performed using the operators $\mathcal{K}$ and $\mathcal{L}$. For $|\chi\rangle$ we derive another result, describing its insertion in a space of surfaces by twist-sewing a three-punctured sphere carrying $|\chi\rangle$ onto each surface of the space. In section 7 we show explicitly how to construct a CFT deformation using the dilaton and $|\chi\rangle$. We then prove that the deformation is actually trivial. We sketch a definition of a string background, and argue that the dilaton deforms it nontrivially. In section 8 we prove the dilaton theorem by constructing an antibracket preserving diffeomorphism of the state space $\widehat{\mathcal{H}}$ into itself, mapping into each other the string field measures $d \mu e^{2 S / \hbar}$ built with two different but nearby values of the dimensionless string coupling constant. The inhomogeneous piece of the diffeomorphism is a shift along the dilaton direction. The nonlinear pieces of the diffeomorphism use both the $\mathcal{B}$ spaces and the state $|\chi\rangle$.

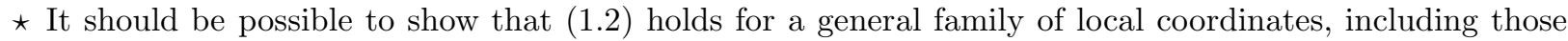
not arising from metrics. We will not attempt to do this in the present work.
} 


\section{Background Material and Preliminary Developments}

This section has three parts. In the first part we review the definition of the ghost-dilaton state $|D\rangle$, introduce the $|\chi\rangle$ state, and give their main properties. This material is taken mostly from Ref. [ 5], and is summarized here for convenience and in order to set up our conventions. In the second part we review the concept of a family of local coordinates. We show explicitly how to extract a family of local coordinates from a conformal metric following Ref.[ 8]. This prescription is familiar in the mathematical literature and applies to any Kähler metric [9]. Finally, in the third part, comprising sections 2.3-2.5, we review the BV algebra of Riemann surfaces, the construction of forms on moduli spaces, and the map from spaces of surfaces to string functionals.

\subsection{Basics OF the Dilaton}

In bosonic string theory formulated on a background spacetime which is twenty six dimensional Minkowski space, the dilaton vertex operator reads

$$
D_{p}(z, \bar{z})=\left(c \bar{c} \partial X \cdot \bar{\partial} X-\frac{1}{2}\left(c \partial^{2} c-\bar{c} \bar{\partial}^{2} \bar{c}\right)\right) e^{i p X}
$$

This state is well-known to be BRST invariant if the on-shell condition $p^{2}=0$ holds. Therefore the dilaton represents a physical massless scalar. Note that the ghost structure of the operator is rather nontrivial. Despite being physical, this operator is not primary. Indeed the operator product expansion with the holomorphic part of the total stress tensor is given by

$$
T(z) D_{p}(w, \bar{w}) \sim \frac{1}{(z-w)^{3}}(-i c \bar{c} p \cdot \bar{\partial} X+c \partial c) e^{i p X}+\cdots
$$

While this only establishes that the above representative of the dilaton BRST cohomology class is not primary, it is also known that there is no primary representative valid for all values of the momentum satisfying $p^{2}=0$. Primary representatives for the dilaton have difficulties with the value $p=0$. The above representative for the dilaton is no exception and is not primary when $p=0$. In this case the violation of the primary state condition arises from the ghosts. It is therefore of interest to focus on the ghost part of the dilaton vertex operator. The

\footnotetext{
$\star$ For other relevant work on dilatons see Refs.[11,12,13] .
} 
zero-momentum ghost-dilaton is present for any consistent string background, and is defined by

$$
D(z, \bar{z}) \equiv \frac{1}{2}\left(c \partial^{2} c-\bar{c}^{2} \bar{c}\right)
$$

or in the Fock space version, by

$$
|D\rangle=\left(c_{1} c_{-1}-\bar{c}_{1} \bar{c}_{-1}\right)|0\rangle
$$

This state is still annihilated by the total BRST operator $Q$. An instructive way to verify this goes as follows. One first checks that

$$
D(z, \bar{z})=\frac{1}{2}\{Q, \partial c-\bar{\partial} \bar{c}\}
$$

and therefore one can write

$$
|D\rangle=\frac{1}{2}\{Q, \partial c-\bar{\partial} \bar{c}\}|0\rangle=Q c_{0}^{-}|0\rangle=-Q|\chi\rangle,
$$

where we have introduced the state $|\chi\rangle$ defined as

$$
|\chi\rangle \equiv-c_{0}^{-}|0\rangle=-\frac{1}{2}\left(c_{0}-\bar{c}_{0}\right)|0\rangle
$$

The representation of $|D\rangle$ in (2.6) makes it manifest that $Q|D\rangle=0$. In addition, the dilaton is annihilated by $b_{0}^{-}\left(\equiv b_{0}-\bar{b}_{0}\right)$

$$
b_{0}^{-}|D\rangle=b_{0}^{-} Q c_{0}^{-}|0\rangle=L_{0}^{-} c_{0}^{-}|0\rangle-Q\left\{b_{0}^{-}, c_{0}^{-}\right\}|0\rangle=0
$$

and is therefore an element of the restricted state space defined by

$$
\widehat{\mathcal{H}}=\left\{|\Psi\rangle \in \mathcal{H}: b_{0}^{-}, L_{0}^{-}|\Psi\rangle=0\right\}
$$

making it a perfectly acceptable physical state of the closed string spectrum. The zeromomentum ghost dilaton is a trivial state in the absolute BRST cohomology, but is not trivial in the semirelative BRST cohomology that properly defines the closed string physical states. This is so because $|D\rangle$ is given by $Q$ acting on the state $|\chi\rangle$, which is not annihilated by $b_{0}^{-}$,

$$
b_{0}^{-}|\chi\rangle=-|0\rangle \neq 0
$$

and therefore, is not part of the restricted state space $\widehat{\mathcal{H}}$. 
As previously stated, the dilaton is not primary, but as expected from (2.2) only $L_{1}$ and $\bar{L}_{1}$ fail to annihilate it

$$
\left\{L_{0}, L_{2}, L_{3}, \ldots\right\}|D\rangle=0, \quad\left\{\bar{L}_{0}, \bar{L}_{2}, \bar{L}_{3}, \ldots\right\}|D\rangle=0
$$

while

$$
L_{1}|D\rangle \neq 0, \quad \text { and } \quad \bar{L}_{1}|D\rangle \neq 0
$$

Indeed,

$$
\begin{aligned}
L_{1}|D\rangle=\left\{Q, b_{1}\right\}|D\rangle & =Q b_{1}\left(c_{1} c_{-1}-\bar{c}_{1} \bar{c}_{-1}\right)|0\rangle \\
& =-Q c_{1}|0\rangle=-\{Q, c\}|0\rangle=-c \partial c|0\rangle=c_{0} c_{1}|0\rangle
\end{aligned}
$$

and similarly $\bar{L}_{1}|D\rangle=-\bar{c}_{0} \bar{c}_{1}|0\rangle$. The $|\chi\rangle$ state is also not primary, it fails to be so just as much as the dilaton

$$
\left\{L_{0}, L_{2}, L_{3}, \ldots\right\}|\chi\rangle=0, \quad\left\{\bar{L}_{0}, \bar{L}_{2}, \bar{L}_{3}, \ldots\right\}|\chi\rangle=0
$$

while

$$
L_{1}|\chi\rangle \neq 0, \quad \text { and } \quad \bar{L}_{1}|\chi\rangle \neq 0 \text {. }
$$

Indeed, one easily verifies that

$$
L_{1}|\chi\rangle=\left\{Q, b_{1}\right\}|\chi\rangle=b_{1} Q|\chi\rangle=-b_{1}|D\rangle=c_{1}|0\rangle
$$

and, similarly, $\bar{L}_{1}|\chi\rangle=-\bar{c}_{1}|0\rangle$.

The representative we have chosen for the zero-momentum dilaton state is not unique. We could have written, for example, $|D\rangle_{\alpha}=-Q c_{0}^{-}|0\rangle+\alpha Q c_{0}^{+}|0\rangle$, with $\alpha$ an arbitrary constant. The second term here represents a truly BRST trivial term. We will find no use in the present work for these representatives and we will use the representative $|D\rangle$ throughout. 


\subsection{Family of local Coordinates from a Conformal Metric}

A family of local coordinates defined over a region $R$ of a Riemann surface is a prescription that assigns a local coordinate around every point in the region $R$. In other words, for each point $p \in R$ we know how to fix a canonical disk around it. We assume we have a uniformizer $z$ for the region $R$, and the region is itself parameterized by two real parameters $\lambda_{1}$ and $\lambda_{2}$. This means that a point $p \in R$ defines uniquely the values of $\lambda_{1}$ and $\lambda_{2}$, and there is a function $z\left(\lambda_{1}, \lambda_{2}\right)=z(p)$. Having a family of local coordinates amounts to having a family of maps $h_{\lambda_{1}, \lambda_{2}}: D \rightarrow R$, taking a unit disk $D$ into the region $R$, such that the center of $D$ is mapped to the point $p \in R$ corresponding to the parameters $\lambda_{1}$ and $\lambda_{2}$ (see Figure 1). We can describe explicitly the family of local coordinates as

$$
z=h_{\lambda_{1} \lambda_{2}}(w)=z\left(\lambda_{1}, \lambda_{2}\right)+a\left(\lambda_{1}, \lambda_{2}\right) w+\frac{1}{2} b\left(\lambda_{1}, \lambda_{2}\right) w^{2}+\frac{1}{3 !} c\left(\lambda_{1}, \lambda_{2}\right) w^{3}+\cdots,
$$

where $w \in D$. The origin of the disk is mapped to the point $p$ specified by the parameters $\lambda_{1}, \lambda_{2}$ because $h_{\lambda_{1} \lambda_{2}}(0)=z\left(\lambda_{1}, \lambda_{2}\right)=z(p)$. Equation (2.16) describes the most general family of local coordinates in the region $R$. This family is specified by the functions $z\left(\lambda_{1}, \lambda_{2}\right), a\left(\lambda_{1}, \lambda_{2}\right), b\left(\lambda_{1}, \lambda_{2}\right)$, and so on.

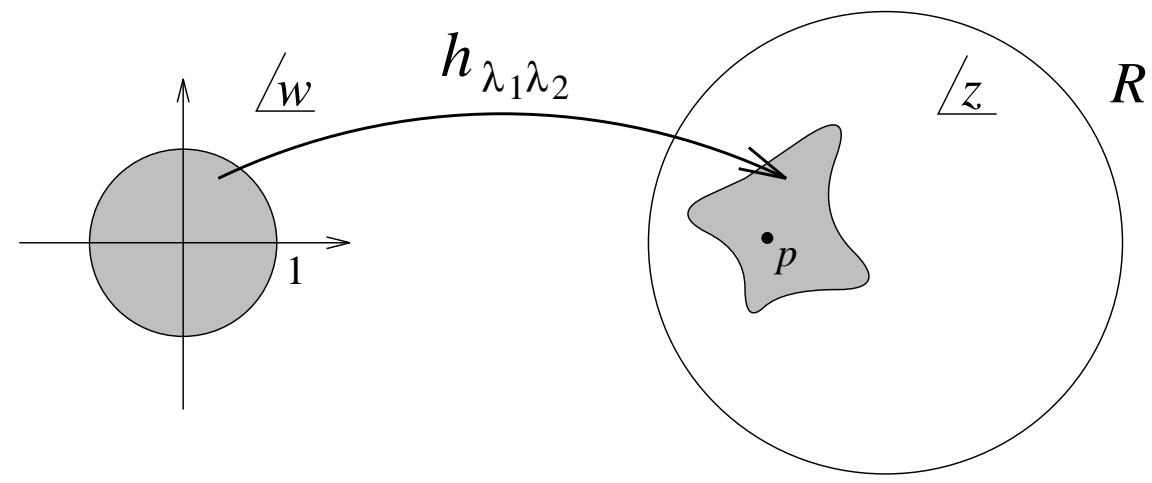

Figure 1. The point $p \in R$ is parameterized by $\lambda_{1}, \lambda_{2}$. The map $h_{\lambda_{1}, \lambda_{2}}$ defines a local coordinate around the point $p$, by mapping the unit disk to some neighborhood of $p$ such that the origin of the disk is mapped to $p$.

Given a metric $\rho(z, \bar{z})$ in a domain $R$ described by the local uniformizer $z$, one can extract in some canonical fashion a family of local coordinates for the domain $R$. One method was proposed in Ref.[7]: the coordinate disk around each point $p$ is defined to be the locus of all points whose shortest distance to $p$ is less than or equal to some fixed number $a_{0}$. While 
this prescription is fairly intuitive it does not lend itself so easily to explicit computation. We will use a related prescription given in Ref.[8]. Fix a point $p \in R$ and let the function $z=h_{\lambda_{1} \lambda_{2}}(w)$, with $z(p)=h_{\lambda_{1} \lambda_{2}}(w(p))=h_{\lambda_{1} \lambda_{2}}(0)$, define the local coordinate $w$ to be used for the point $p$. In this prescription $h_{\lambda_{1} \lambda_{2}}(w)$ is determined by pulling back the metric to the $w$ plane, and demanding that the pulled metric $\rho^{w}$ satisfy $\left.\partial_{w}^{n} \rho^{w}\right|_{w=0}=\left.\partial_{\bar{w}}^{n} \rho^{w}\right|_{w=0}=0$, for all positive values of $n$. This prescription, of course, does not determine the parameterization $z\left(\lambda_{1}, \lambda_{2}\right)$ which must be supplied. It also does not determine the first coefficient $a\left(\lambda_{1}, \lambda_{2}\right)$ of the series expansion. The absolute value $|a|$ can be fixed easily, but the phase of $a$ cannot be fixed in any straightforward way. All higher order coefficients are uniquely determined.

We begin our computation by relating the metric $\rho^{z}$ referred to the uniformizer $z$ to the metric $\rho^{w}$ referred to the $w$ coordinate,

$$
\rho^{z}|d z|=\rho^{w}|d w|, \quad \rightarrow \quad \rho^{w}=\rho^{z}\left|\frac{d h_{\lambda}}{d w}\right| .
$$

Our aim is to use the above equation to express the metric in the $w$ plane as a power series in $w$ and $\bar{w}$. Expanding the right hand side we find

$$
\rho^{w}=\left[\left.\rho^{z}\right|_{p}+\left.(z-z(p)) \partial_{z} \rho^{z}\right|_{p}+\left.(\bar{z}-\overline{z(p)}) \partial_{\bar{z}} \rho^{z}\right|_{p}+\cdots\right] \cdot|a+b w+\cdots|,
$$

where we only keep terms linear in $w$. The coordinate difference $(z-z(p))$ can be expressed in terms of $w$ using the definition of the family of local coordinates $(2.16)$,

$$
\rho^{w}=\left[\left.\rho^{z}\right|_{p}+\left.a w \partial_{z} \rho^{z}\right|_{p}+\left.\bar{a} \bar{w} \partial_{\bar{z}} \rho^{z}\right|_{p}+\cdots\right]|a|\left(1+\frac{b}{2 a} w+\frac{\bar{b}}{2 \bar{a}} \bar{w}+\cdots\right) .
$$

Collecting all terms of order $w($ or $\bar{w})$ we find

$$
\rho^{w}=\left.|a| \rho^{z}\right|_{p}\left[1+a w\left(\left.\partial_{z} \ln \rho^{z}\right|_{p}+\frac{b}{2 a^{2}}\right)+\bar{a} \bar{w}\left(\left.\partial_{\bar{z}} \ln \rho^{z}\right|_{p}+\frac{\bar{b}}{2 \bar{a}^{2}}\right)+\cdots\right] .
$$

The prescription now requires that $\left.\partial_{w} \rho^{w}\right|_{w=0}=\left.\partial_{\bar{w}} \rho^{w}\right|_{w=0}=0$, giving us

$$
\frac{b}{2 a^{2}}=-\left.\partial_{z} \ln \rho^{z}\right|_{p}, \quad \text { and } \quad \frac{\bar{b}}{2 \bar{a}^{2}}=-\left.\partial_{\bar{z}} \ln \rho^{z}\right|_{p}
$$

Since these equations (consistent with complex conjugation, since $\rho$ is real) determine the ratio $b / a^{2}$ for each point $p \in R$ we can simply write

$$
\frac{b}{2 a^{2}}=-\partial_{z} \ln \rho^{z}
$$

This equation will be quite useful later on. As mentioned earlier, $|a|$ can be fixed naturally by setting $|a|=1 /\left.\rho^{z}\right|_{p}$. In this way the metric $\rho^{w}$ becomes of the form $\rho^{w}=1+\cdots$, as can be 
seen from (2.20). The procedure does not fix the phase of $a$ over the region $R$. We will learn how to fix that phase in a natural way for the case of families of local coordinates over curves.

\subsection{Algebra of Riemann Surfaces}

We review in this section some of the geometrical results of Ref. [7]. The following spaces of Riemann surfaces will be of use to us : $\mathcal{M}_{g, n}$ denotes the usual moduli space of Riemann surfaces of genus $g$ and $n$ punctures, $\overline{\mathcal{M}}_{g, n}$ denotes its compactification (which includes the degenerate surfaces), $\mathcal{P}_{g, n}$ denotes the space of Riemann surfaces of genus $g$ and $n$ punctures with local coordinates defined at each puncture, and $\widehat{\mathcal{P}}_{g, n}$ is the same as $\mathcal{P}_{g, n}$ except that the phases of the local coordinates are not specified. The spaces $\mathcal{P}_{g, n}$ and $\widehat{\mathcal{P}}_{g, n}$ are fiber bundles over $\overline{\mathcal{M}}_{g, n}$, and $\mathcal{P}_{g, n}$ is a circle bundle over $\widehat{\mathcal{P}}_{g, n}$. We will be dealing mostly with $\widehat{\mathcal{P}}_{g, n}$, since this is the space that is directly relevant in string field theory.

Algebra of Riemann Surfaces Local coordinates allow us to twist-sew two punctures by identifying points whose local coordinates are related by

$$
z_{1} z_{2}=t
$$

where $t=\exp (i \theta)$, with $\theta \in[0,2 \pi]$. Given two symmetric ${ }^{\star}$ subspaces $\mathcal{A}_{1} \subset \widehat{\mathcal{P}}_{g_{1}, n_{1}}$ and $\mathcal{A}_{2} \subset \widehat{\mathcal{P}}_{g_{2}, n_{2}}$, the subspace $\left\{\mathcal{A}_{1}, \mathcal{A}_{2}\right\} \subset \widehat{\mathcal{P}}_{g_{1}+g_{2}, n_{1}+n_{2}-2}$ is defined by twist sewing every surface in $\mathcal{A}_{1}$ to every surface in $\mathcal{A}_{2}$, and symmetrizing over the $\left(n_{1}+n_{2}-2\right)$ remaining punctures. We can use any two fixed (labeled) punctures to sew since the subspaces are symmetric. This operation possesses the following properties

$$
\begin{gathered}
\left\{\mathcal{A}_{1}, \mathcal{A}_{2}\right\}=-(-)^{\left(\mathcal{A}_{1}+1\right)\left(\mathcal{A}_{2}+1\right)}\left\{\mathcal{A}_{2}, \mathcal{A}_{1}\right\}, \\
(-)^{\left(A_{1}+1\right)\left(A_{3}+1\right)}\left\{\left\{\mathcal{A}_{1}, \mathcal{A}_{2}\right\}, \mathcal{A}_{3}\right\}+\text { cyclic }=0, \\
\partial\left\{\mathcal{A}_{1}, \mathcal{A}_{2}\right\}=\left\{\partial \mathcal{A}_{1}, \mathcal{A}_{2}\right\}+(-)^{\mathcal{A}_{1}+1}\left\{\mathcal{A}_{1}, \partial \mathcal{A}_{2}\right\},
\end{gathered}
$$

where $\mathcal{A}_{1}$ and $\mathcal{A}_{2}$ in the exponents denote the dimensions of the spaces. Given a symmetric subspace $\mathcal{A} \subset \widehat{\mathcal{P}}_{g, n}$, the subspace $\Delta \mathcal{A} \subset \widehat{\mathcal{P}}_{g+1, n-2}$ is defined by twist sewing two fixed (labeled)

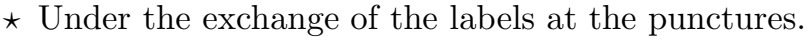


punctures on every surface in $\mathcal{A}$, and multiplying by a factor of a half. Together with the antibracket defined above this operation satisfies

$$
\begin{aligned}
\Delta^{2} \mathcal{A} & =0 \\
\Delta\left\{\mathcal{A}_{1}, \mathcal{A}_{2}\right\} & =\left\{\Delta \mathcal{A}_{1}, \mathcal{A}_{2}\right\}+(-)^{\mathcal{A}_{1}+1}\left\{\mathcal{A}_{1}, \Delta \mathcal{A}_{2}\right\} \\
\Delta \partial \mathcal{A} & =-\partial \Delta \mathcal{A} .
\end{aligned}
$$

If one defines a complex $\widehat{\mathcal{P}}$ as the formal sum, over $g$ and $n$, of the spaces $\widehat{\mathcal{P}}_{g, n}$, the antibracket and delta operator are well defined in this complex.

Occasionally we will underline a symmetric subspace $\underline{\mathcal{A}}$ to denote that one of its punctures has been selected as a special puncture. Since the space is symmetric, the choice of puncture is irrelevant. This puncture is reserved for the insertion of some definite external state, and is therefore never sewn. The subspaces $\{\underline{\mathcal{A}}, \mathcal{B}\}$ and $\Delta \underline{\mathcal{A}}$ are then defined as above except that the special puncture is ignored altogether. For the antibracket, symmetrization is performed only over the ordinary punctures, with the special puncture fixed on $\underline{\mathcal{A}}$. Consequently $\{\underline{\mathcal{A}}, \mathcal{B}\}$ is only symmetric under the exchange of ordinary punctures.

\subsection{Forms on Moduli Spaces}

The operator formalism of CFT assigns a state $\langle\Sigma| \in\left(\mathcal{H}^{*}\right)^{\otimes n}$ to every surface $\Sigma \in \mathcal{P}_{g, n}$. This allows one to define canonical forms in $T_{\Sigma}\left(\mathcal{P}_{g, n}\right)$ by the rule ${ }^{\dagger}$

$$
\left\langle\Omega^{[k] g, n}\right|\left(V_{1}, \cdots, V_{k}\right) \equiv(-2 \pi i)^{(3-n-3 g)}\langle\Sigma| \mathbf{b}\left(\mathbf{v}_{1}\right) \cdots \mathbf{b}\left(\mathbf{v}_{k}\right)
$$

The Schiffer vector $\mathbf{v}_{r}=\left(v_{r}^{(1)}(z), \cdots v_{r}^{(n)}(z)\right)$ creates the deformation of the surface $\Sigma$ specified by the tangent $V_{r}$, and the antighost insertions are given by $(\oint d z / z=\oint d \bar{z} / \bar{z}=2 \pi i)$

$$
\mathbf{b}(\mathbf{v})=\sum_{i=1}^{n}\left(\oint b^{(i)}\left(z_{i}\right) v^{(i)}\left(z_{i}\right) \frac{d z_{i}}{2 \pi i}+\oint \bar{b}^{(i)}\left(\bar{z}_{i}\right) \bar{v}^{(i)}\left(\bar{z}_{i}\right) \frac{d \bar{z}_{i}}{2 \pi i}\right) .
$$

These forms satisfy the familiar $Q \leftrightarrow d$ correspondence,

$$
\left\langle\Omega^{[k] g, n}\right| \sum_{i=1}^{n} Q^{(i)}=(-1)^{k} d\left\langle\Omega^{[k-1] g, n}\right| .
$$

When the states inserted at the $n$ punctures are in $\widehat{\mathcal{H}}$ these forms descend to well-defined forms on $T_{\Sigma} \widehat{\mathcal{P}}_{g, n}[11,14]$.

$\dagger$ The notation differs slightly from reference [7] in the way the degree of the form is specified. In [7] $\Omega^{(k) g, n}$ denoted a $(6 g-6+2 n+k)$-form, here $\Omega^{[k] g, n}$ will denote a $k$-form. We changed the rounded parentheses to brackets to eliminate possible confusion, if both notations were to be used simultaneously. 
We now develop an application of the above results that will be useful in our later developments. We will calculate the explicit antighost insertions associated with tangents that represent the motion of a puncture in a domain $R$ of some surface. As the puncture moves the local coordinate at that puncture changes. We will also assume that as this motion takes place the rest of the surface data (moduli, position of the other punctures, and their local coordinates) does not change. Therefore the Schiffer vector will be supported only on the moving puncture. The associated antighost insertion will be necessary to study the effect of insertions of $|D\rangle$ and $|\chi\rangle$ in correlators.

The local coordinate at the moving puncture is described by the family of local coordinates (2.16) over a region $R$ of a Riemann surface (with uniformizer $z$ ). Associated with the real parameter $\lambda_{i}$ there is a Schiffer vector $v\left(\lambda_{i}\right)$ given by [10]

$$
v_{\lambda_{i}}(w)=-\frac{d h_{\lambda_{1} \lambda_{2}}(w)}{d \lambda_{i}}\left[\frac{d h_{\lambda_{1} \lambda_{2}}(w)}{d w}\right]^{-1} .
$$

Evaluation gives

$$
v_{\lambda_{i}}(w)=\alpha_{\lambda_{i}}+\beta_{\lambda_{i}} w+\gamma_{\lambda_{i}} w^{2}+\cdots
$$

where the first three coefficients are given by

$$
\begin{aligned}
& \alpha_{\lambda_{i}}=-\frac{1}{a} \frac{d z}{d \lambda_{i}} \\
& \beta_{\lambda_{i}}=-\frac{1}{a} \frac{d a}{d \lambda_{i}}+\frac{b}{a^{2}} \frac{d z}{d \lambda_{i}} \\
& \gamma_{\lambda_{i}}=-a \frac{d}{d \lambda_{i}}\left(\frac{b}{2 a^{2}}\right)-\left(\frac{b^{2}}{a^{3}}-\frac{1}{2} \frac{c}{a^{2}}\right) \frac{d z}{d \lambda_{i}}
\end{aligned}
$$

The antighost insertion corresponding to this Schiffer vector is then

$$
\begin{aligned}
\mathbf{b}\left(v_{\lambda_{i}}\right)= & \alpha_{\lambda_{i}} b_{-1}+\beta_{\lambda_{i}} b_{0}+\gamma_{\lambda_{i}} b_{1}+\cdots \\
& +\bar{\alpha}_{\lambda_{i}} \bar{b}_{-1}+\bar{\beta}_{\lambda_{i}} \bar{b}_{0}+\bar{\gamma}_{\lambda_{i}} \bar{b}_{1}+\cdots
\end{aligned}
$$

where the bars denote complex conjugation on the coefficients, and antiholomorphic sector on the antighosts. 


\subsection{Maps to String Functionals}

Integration of the the canonical forms over subspaces of $\widehat{\mathcal{P}}_{g, n}$ defines a map from these subspaces to functions on the vector space $\widehat{\mathcal{H}}$. One takes

$$
f\left(\mathcal{A}_{g, n}^{[k]}\right) \equiv \frac{1}{n !} \int_{\mathcal{A}_{g, n}^{[k]}}\left\langle\Omega^{[k] g, n} \mid \Psi\right\rangle_{1} \cdots|\Psi\rangle_{n}, \quad n \geq 1,
$$

where $\mathcal{A}_{g, n}^{[k]}$ is a $k$-dimensional subspace, and the subscripts on the string field insertions denote which copy of $\widehat{\mathcal{H}}$ they belong to. In the space $\widehat{\mathcal{H}}$ there is a natural symplectic structure. The antibracket and delta operator are given by $[7]$

$$
\begin{aligned}
\{f, g\} & =(-)^{g+1} \frac{\partial f}{\partial|\Psi\rangle} \frac{\partial g}{\partial|\Psi\rangle}|\mathcal{S}\rangle, \\
\Delta f & =(-)^{f+1}\left(\frac{\partial}{\partial|\Psi\rangle} \frac{\partial}{\partial|\Psi\rangle} f\right)|\mathcal{S}\rangle,
\end{aligned}
$$

where $(-)^{g}$ denotes the grassmanality of $g$, and the twist-sewing ket $|\mathcal{S}\rangle \in \widehat{\mathcal{H}}^{\otimes 2}$ is given by

$$
\left|\mathcal{S}_{12}\right\rangle=b_{0}^{-(1)}\left|R_{12}^{\prime}\right\rangle=b_{0}^{-(1)} \int \frac{d \theta}{2 \pi} e^{i \theta L_{0}^{-(1)}}\left|R_{12}\right\rangle,
$$

where the reflector ket $\left|R_{12}\right\rangle$ is defined by $\left\langle R_{12} \mid R_{23}\right\rangle={ }_{3} \mathbf{1}_{1}$. The twist-sewing ket satisfies $\left\langle\omega_{12} \mid \mathcal{S}_{23}\right\rangle={ }_{3} \mathbf{1}_{1}$, where $\left\langle\omega_{12}\right| \equiv\left\langle R_{12}^{\prime}\right| c_{0}^{-(2)}$ is the symplectic form in $\widehat{\mathcal{H}}$.

It then follows that for $\mathcal{A}, \mathcal{B} \subset \widehat{\mathcal{P}}$ the following representation identities hold [7],

$$
\begin{aligned}
f(\Delta \mathcal{A}) & =-\Delta f(\mathcal{A}) \\
f(\{\mathcal{A}, \mathcal{B}\}) & =-\{f(\mathcal{A}), f(\mathcal{B})\} .
\end{aligned}
$$

The above equations define a homomorphism from the BV algebra on subspaces of $\widehat{\mathcal{P}}$ to a $\mathrm{BV}$ algebra on string field functionals in $\widehat{\mathcal{H}}$, a fact that is formulated in a precise fashion in Ref.[ 15]. From (2.29) it follows that

$$
\left\{S_{0,2}, f(\mathcal{A})\right\}=-f(\partial \mathcal{A}),
$$

where $S_{0,2}$ is the kinetic term of the string field action, given by

$$
S_{0,2}=\frac{1}{2}\left\langle\Psi\left|c_{0}^{-} Q\right| \Psi\right\rangle=\frac{1}{2}\left\langle\omega_{12}\left|Q^{(2)}\right| \Psi\right\rangle_{1}|\Psi\rangle_{2} .
$$

Maps with Insertions. More general maps can be defined with the use of specific states in the state space of the conformal theory. We will denote by $|\mathcal{O}\rangle$ states in $\mathcal{H}$ and by $|\widehat{\mathcal{O}}\rangle$ states in the 
restricted space $\widehat{\mathcal{H}}$. The state may have arbitrary grassmanality and arbitrary ghost number. We will only consider functionals with one additional insertion, defined as

$$
f_{\mathcal{O}}\left(\mathcal{A}_{g, n+1}^{[k]}\right) \equiv \frac{1}{n !} \int_{\mathcal{A}_{g, n+1}^{[k]}}\left\langle\Omega^{[k] g, n+1} \mid \Psi\right\rangle_{1} \cdots|\Psi\rangle_{n}|\mathcal{O}\rangle_{n+1}
$$

and similarly for states $|\widehat{\mathcal{O}}\rangle$. If $\mathcal{A}$ is a symmetric space the state can be inserted anywhere, but if there is one puncture that is not symmetrized over the state must be inserted at that puncture. For general subspaces of $\widehat{\mathcal{P}}$ we must only use $|\widehat{\mathcal{O}}\rangle$ states. This condition is well known to make the insertions well-defined regardless of the phase of the local coordinate at the insertion point. This is important because typically there are no sections in $\mathcal{P}_{g, n}$ over general subspaces of $\widehat{\mathcal{P}}_{g, n}$, namely, one cannot fix the phases of the local coordinates smoothly over general subspaces of $\widehat{\mathcal{P}}_{g, n}$. However, in subspaces over which the phase of the local coordinate at the special puncture can be defined continuously, we can insert $|\mathcal{O}\rangle$ states without ambiguities. In the following section we will introduce a large class of such subspaces, the simplest of which are spaces of three-punctured spheres. These spaces will later be used to insert the unrestricted state $|\chi\rangle$.

The following identities can be shown to hold

$$
\begin{aligned}
& f_{\mathcal{O}}(\Delta \mathcal{A})=-\Delta f_{\mathcal{O}}(\mathcal{A}), \\
& f_{\mathcal{O}}(\{\mathcal{A}, \underline{\mathcal{B}}\})=-\left\{f(\mathcal{A}), f_{\mathcal{O}}(\mathcal{B})\right\}, \\
& \left\{S_{0,2}, f_{\mathcal{O}}(\mathcal{A})\right\}=-f_{\mathcal{O}}(\partial \mathcal{A})+(-)^{\mathcal{A}} f_{Q \mathcal{O}}(\mathcal{A}) \text {, }
\end{aligned}
$$

where the underline denotes the space in which the extra state is inserted. The first two identities were given in [7], for the case of grassmann even states in $\widehat{\mathcal{H}}$. Note that the special puncture in the surfaces contained in the $\mathcal{A}$ and $\mathcal{B}$ spaces must have a well defined phase. For each of the above equations there are trivial cases. If the surfaces in $\mathcal{A}$ have less than three punctures the first equation gives zero equal zero. If the surfaces in $\mathcal{A}$ have no punctures, or those in $\mathcal{B}$ have less than two punctures, the second equation is trivial. Finally, if the surfaces in $\mathcal{A}$ have only one puncture the left hand side of the third equation vanishes identically, and the right hand side vanishes by cancelation,

$$
f_{\mathcal{O}}\left(\partial \mathcal{A}_{g, 1}\right)=(-)^{\mathcal{A}_{g, 1}} f_{Q \mathcal{O}}\left(\mathcal{A}_{g, 1}\right)
$$

It is useful to introduce, for states in $\widehat{\mathcal{H}}$, the odd Hamiltonian function,

$$
\mathbf{U}_{\widehat{\mathcal{O}}(0,2)} \equiv\left\langle\omega_{12} \mid \widehat{\mathcal{O}}\right\rangle_{1}|\Psi\rangle_{2}
$$

Note that for states $|\mathcal{O}\rangle$ annihilated by $c_{0}^{-}$, this definition would give zero. This function 
implements the insertion of the state $|\widehat{\mathcal{O}}\rangle$ by a canonical transformation,

$$
f_{\widehat{\mathcal{O}}}(\mathcal{A})=\left\{f(\mathcal{A}), \mathbf{U}_{\widehat{\mathcal{O}}(0,2)}\right\}
$$

Furthermore,

$$
\left\{S_{0,2}, \mathbf{U}_{\widehat{\mathcal{O}}(0,2)}\right\}=-\mathbf{U}_{Q \widehat{\mathcal{O}}(0,2)},
$$

which vanishes if the state $|\widehat{\mathcal{O}}\rangle$ is physical. Given that the string action $S$ can be written as

$$
S=S_{0,2}+f(\mathcal{V})+\hbar S_{1,0}
$$

where $S_{1,0}$ is string field independent and $\mathcal{V}$ are the string vertices (to be reviewed briefly in sec. 3.2 ), we find

$$
\left\{S, f_{\mathcal{O}}(\mathcal{A})\right\}=(-)^{\mathcal{A}} f_{Q \mathcal{O}}(\mathcal{A})-f_{\mathcal{O}}(\partial \mathcal{A}+\{\mathcal{V}, \underline{\mathcal{A}}\})
$$

where use was made of the last two equations in (2.41). Notice that if the surfaces in $\mathcal{A}$ have just one puncture the above equation is still valid, and becomes zero equal zero by virtue of (2.42). Again, the special puncture of the surfaces in $\mathcal{A}$ must have a coordinate with a well-defined phase whenever the state $|\mathcal{O}\rangle$ is outside $\widehat{\mathcal{H}}$. Another useful relation follows from (2.44) and (2.45) ,

$$
\left\{S, \mathbf{U}_{\widehat{\mathcal{O}}(0,2)}\right\}=-\mathbf{U}_{Q \widehat{\mathcal{O}}(0,2)}+f_{\widehat{\mathcal{O}}}(\underline{\mathcal{V}})
$$

\section{Adding Punctures and $\mathcal{B}$-spaces}

The present section is an extension of some of the work in Ref.[7]. As far as Riemann surfaces are concerned, the main tools introduced in [7] were an operator $\mathcal{K}$ that inserts a new puncture into surfaces, and a series of spaces $\mathcal{B}$ satisfying geometrical recursion relations. Since Ref.[7] was concerned with deformations by primary fields, there were simplifications that are not possible when we consider the dilaton. We will therefore refine the discussion by introducing several classes of $\mathcal{K}$ operators, a new insertion operator $\mathcal{L}$, and $\mathcal{B}$ spaces with one puncture. 


\subsection{AdDing A PUnCTURE}

An operator $\mathcal{K}$ that adds punctures to Riemann surfaces was defined in [7]. Given a surface $\Sigma \in \widehat{\mathcal{P}}_{g, n}$, we define a two-dimensional subspace $\mathcal{K}(\Sigma) \subset \widehat{\mathcal{P}}_{g, n+1}$ which contains the $(n+1)$-punctured surfaces given by $\Sigma$ with an extra puncture lying anywhere in the region $\Sigma-\cup_{i} D_{i}$, where $D_{i}$ are unit disks around the original punctures. The local coordinate at the extra puncture is fixed arbitrarily but continuously over the relevant region. For a subspace $\mathcal{A} \subset \widehat{\mathcal{P}}_{g, n}$, we define the subspace $\mathcal{K} \mathcal{A} \subset \widehat{\mathcal{P}}_{g, n+1}$ as the collection of spaces $\mathcal{K}(\Sigma)$ for all $\Sigma \in \mathcal{A}$. We will refer to such subspaces as $\mathcal{K}$-spaces. If the subspace $\mathcal{A}$ is symmetric then $\mathcal{K} \mathcal{A}$ will be symmetric in its first $n$ punctures. The inserted puncture is special in that it is not symmetrized over and is never sewn. The arbitrariness in choice of the local coordinate at the special puncture necessitates the introduction of the concept of weak equality. Two subspaces $\mathcal{A}, \mathcal{B} \subset \widehat{\mathcal{P}}_{g, n}$, containing each one special puncture, are said to be weakly equal, $\mathcal{A} \approx \mathcal{B}$, if they are equal up to the local coordinates at the special punctures. One can show that the following weak identities hold [7],

$$
\begin{gathered}
\mathcal{K}\left(\left\{\mathcal{A}_{1}, \mathcal{A}_{2}\right\}\right) \approx\left\{\mathcal{K} \mathcal{A}_{1}, \mathcal{A}_{2}\right\}+\left\{\mathcal{A}_{1}, \mathcal{K} \mathcal{A}_{2}\right\} \\
\mathcal{K}(\Delta \mathcal{A}) \approx \Delta(\mathcal{K} \mathcal{A}) \\
{[\partial, \mathcal{K}] \approx-\left\{\mathcal{V}_{0,3}^{\prime},\right\}}
\end{gathered}
$$

where $\mathcal{V}_{0,3}^{\prime}$ is the 3-punctured sphere with one special puncture introduced in [6]. It is symmetric under the exchange of two of its punctures. The third, special puncture, is the one associated with $\mathcal{K}$. The above weak equalities were sufficient for the discussion of background independence, since the states inserted at the special puncture were always primary. For the dilaton we need strong equalities. This is so because the dilaton is not primary and its insertion depends on the local coordinate at the special puncture.

We can fix the local coordinate at the special puncture using the metric which solves the minimal area problem of closed string field theory. ${ }^{\ddagger}$ Given a surface $\Sigma$ equipped with coordinate disks $D_{i}$ around the punctures, we find the minimal area metric on $\Sigma$ and use the prescription of sec. 2.2 to extract local coordinates at every point on $\Sigma-\cup D_{i}$. These are the coordinates to

$\star$ In [7] this operator was actually denoted as $\widehat{\mathcal{K}}$.

$\dagger$ When we insert states such as $|\chi\rangle$, we must even fix the phase of the local coordinate at the special puncture.

$\ddagger$ The conformal metric of least area under the condition that all nontrivial closed curves be longer than or equal to $2 \pi$. 
be used for inserting the extra puncture, and the corresponding insertion operator is denoted as $\overline{\mathcal{K}}$. Recall that the minimal area metric gives a canonical way of choosing coordinate disks on any punctured surface. If $^{\S}$ the punctured surface $\Sigma$ comes equipped with its own coordinate disks, these may or may not coincide with the coordinate disks that the minimal area metric on $\Sigma$ would determine. It follows that the operation $\overline{\mathcal{K}}$ of adding an extra puncture using the minimal area metric, is particularly natural acting on surfaces whose coordinate curves are chosen using the minimal area metric. For such spaces of surfaces, denoted as $\mathcal{V}_{i}$, we find the strong identities

$$
\begin{aligned}
\overline{\mathcal{K}}\left(\left\{\mathcal{V}_{1}, \mathcal{V}_{2}\right\}\right) & =\left\{\overline{\mathcal{K}} \mathcal{V}_{1}, \mathcal{V}_{2}\right\}+\left\{\mathcal{V}_{1}, \overline{\mathcal{K}} \mathcal{V}_{2}\right\} \\
\overline{\mathcal{K}}\left(\Delta \mathcal{V}_{i}\right) & =\Delta\left(\overline{\mathcal{K}} \mathcal{V}_{i}\right) \\
{[\partial, \overline{\mathcal{K}}] } & =-\left\{\mathcal{V}_{0,3}^{\prime},\right\} .
\end{aligned}
$$

The first two equations hold by virtue of the compatibility of minimal area metrics with the operation of sewing. The last equation holds acting on $\mathcal{V}_{i}$ spaces, when the coordinate at the special puncture of $\mathcal{V}_{0,3}^{\prime}$ is induced by the metric representing the underlying symmetric two punctured sphere as a flat infinite cylinder of circumference $2 \pi$.

As minimal area metrics have curvature singularities, extracting local normal-coordinates can be delicate. One can smooth out the singularities by changing the metric on the surface minus its unit disks, keeping it unaltered in some neighborhood of the unit disks. The deformed metric will still be flat and isometric to that of a cylinder of circumference $2 \pi$ throughout the unit disks. This metric is no longer unique, as there are many ways of deforming the minimal area metric on the surface. The associated insertion operator will be denoted $\mathcal{K}^{*}$. In a sense, this is the operator most appropriate for the dilaton. Note that for these metrics sewing is still isometric gluing since the coordinate curves are still geodesics of the metric. Moreover, the bulk curvature of these metrics on the surface minus its disks is not changed from its original value. As we will see at the end of sect. 6.1, while the first two equations in (3.4) only hold weakly for $\mathcal{K}^{*}$, they will actually hold strongly when a dilaton is inserted at the special puncture. The third equation in (3.4) holds strongly for $\mathcal{K}^{*}$ since it involves only the coordinate curves of the surfaces, where the metric is unaltered.

The $\mathcal{L}$ operator It is convenient to introduce an operator $\mathcal{L}$ that, acting on a surface $\Sigma \in \widehat{\mathcal{P}}_{g, n}$, gives a one-dimensional subspace $\mathcal{L}(\Sigma) \subset \widehat{\mathcal{P}}_{g, n+1}$ which contains the $(n+1)$-punctured surfaces given by $\Sigma$ with an extra puncture lying anywhere on the coordinate curves $\partial\left(\Sigma-\cup D_{i}\right)$. The local coordinate at the extra puncture is fixed arbitrarily but continuously on the curves. For a

$\S$ For such a metric some neighborhood of each puncture is isometric to a semi-infinite cylinder of circumference $2 \pi$. The coordinate curve is chosen to be the geodesic on the cylinder a distance $\pi$ from the boundary of the cylinder. 
subspace $\mathcal{A} \subset \widehat{\mathcal{P}}_{g, n}$, we define the subspace $\mathcal{L} \mathcal{A} \subset \widehat{\mathcal{P}}_{g, n+1}$ as the collection of spaces $\mathcal{L}(\Sigma)$ for all $\Sigma \in \mathcal{A}$. We will refer to such subspaces as $\mathcal{L}$-spaces. If the subspace $\mathcal{A}$ is symmetric then $\mathcal{L} \mathcal{A}$ will be symmetric in its first $n$ punctures. The orientation of $\mathcal{L} \mathcal{A}$ is defined as follows. Let $\{\mathcal{A}\}$ denote the orientation of $\mathcal{A}$ at some point corresponding to the surface $\Sigma$, and let the special puncture be at some point on one of the coordinate curves. Let $v$ denote the tangent vector to the coordinate curve at that point, with the coordinate curve oriented as the boundary of $\Sigma-D$, and let $\widehat{V}(v)$ be the corresponding tangent in $\widehat{\mathcal{P}}_{g, n+1}$ representing the motion of the special puncture. The orientation of $\mathcal{L} \mathcal{A}$ is then defined as $[\widehat{V}(v),\{\mathcal{A}\}]$. For more details the reader may consult sect. 2.3 of Ref.[ 7$]$.

The definition of $\mathcal{L}$ helps us clarify Eqn. (3.3). Just by virtue of our definitions, the commutator of the boundary operator $\partial$ and $\mathcal{K}$ is precisely $\mathcal{L}$,

$$
[\partial, \mathcal{K}]=\mathcal{L}
$$

as one readily verifies by acting on a space of surfaces. It then follows from (3.3) that $\mathcal{L} \approx$ $-\left\{\mathcal{V}_{0,3}^{\prime},\right\}$. If we use a particular metric on the surfaces of a space $\mathcal{A}$ to define the local coordinates for the insertion $\mathcal{K}$ we will use the same metric for the insertion $\mathcal{L}$. It then follows that $\overline{\mathcal{L}}$, defined using the minimal area metric, satisfies

$$
[\partial, \overline{\mathcal{K}}]=\overline{\mathcal{L}}, \quad \overline{\mathcal{L}}=-\left\{\mathcal{V}_{0,3}^{\prime},\right\}
$$

where the second equation holds acting on $\mathcal{V}_{i}$ spaces by virtue of the last equation in (3.4).

If the metric used by $\mathcal{L}$ to extract the local coordinate at the special puncture is invariant under rigid $U(1)$ rotations of the coordinate curve, we expect that the $\mathcal{L}$ insertion can be reproduced by twist-sewing a three-punctured sphere $\mathcal{V}_{0,3}^{*}$ whose local coordinate at the special puncture is chosen to match the one used by $\mathcal{L}$. If the metric used by $\mathcal{L}$ is not $U(1)$ invariant there is no guarantee that the $\mathcal{L}$ space can be obtained by twist sewing.

$\mathcal{L}$ spaces are useful since one can define the phase of the local coordinate at the special puncture uniquely for each surface $\Sigma \in \mathcal{L} \mathcal{A}$, and continuously over the space $\mathcal{L} \mathcal{A}$. This does not quite define a section in $\mathcal{P}$, since we are only fixing the phase at the special puncture, but it does allow the insertion of an unrestricted state (like $|\chi\rangle$ ) at the special puncture. The phase is defined by identifying it with the direction of the tangent to the coordinate curve where the special puncture is inserted. Continuity over $\mathcal{L} \mathcal{A}$ is is guaranteed by uniqueness, and the continuity of the coordinate curves over $\mathcal{A}$. 


\subsection{The Homotopy Spaces $\mathcal{B}$}

The string vertices of string field theory $\mathcal{V}_{g, n}$ are sections in $\widehat{\mathcal{P}}_{g, n}$ over compact subsets of the moduli spaces $\mathcal{M}_{g, n}$ [14]. They are all assembled together as

$$
\mathcal{V} \equiv \sum_{g, n} \hbar^{g} \kappa^{2 g-2+n} \mathcal{V}_{g, n}, \quad \text { with } \quad\left\{\begin{array}{l}
n \geq 3 \text { for } g=0 \\
n \geq 1 \text { for } g=1 \\
n \geq 0 \text { for } g \geq 2
\end{array}\right.
$$

Note that vacuum vertices $\mathcal{V}_{g, 0}$ exist for $g \geq 2$, but not for genus one. We have also included in the sum above the relevant powers of the string field coupling constant $\kappa$ (set equal to one in [7]). The string vertices satisfy the following recursion relations

$$
\partial \mathcal{V}+\hbar \Delta \mathcal{V}+\frac{1}{2}\{\mathcal{V}, \mathcal{V}\}=0
$$

A comment is in order here. While the vacuum vertices are not relevant to the master equation, they are relevant to the action. The recursion relations (3.8), originally written only for vertices with punctures [16], were later recognized [14] to determine the vacuum vertices, since the operations $\Delta \mathcal{V}$ and $\{\mathcal{V}, \mathcal{V}\}$ produce surfaces without punctures even if all the surfaces in $\mathcal{V}$ had punctures. Thus (3.8) gives constraints for vacuum vertices. Since the operations $\Delta \mathcal{V}$ and $\{\mathcal{V}, \mathcal{V}\}$ cannot produce surfaces of genus one without punctures (recall that at genus zero the lowest vertex has three punctures) it is not natural to include a vacuum vertex $\mathcal{V}_{1,0}{ }^{\star}$

A set of subspaces $\mathcal{B}_{g, n} \subset \widehat{\mathcal{P}}_{g, n}$ of dimension $(6 g+2 n-6)+1$ were introduced in [ 7]:

$$
\mathcal{B} \equiv \sum_{g, n} \hbar^{g} \kappa^{2 g-2+n} \mathcal{B}_{g, n} \quad \text { with } \quad\left\{\begin{array}{l}
n \geq 3 \text { for } g=0 \\
n \geq 2 \text { for } g \geq 1
\end{array}\right.
$$

Each space $\mathcal{B}_{g, n}$ must have one special puncture, and is symmetric in all others. The marginal operator, or the dilaton in this paper, is inserted at the special puncture. The string field is to be inserted at the other punctures. The requirement that $\mathcal{B}$ spaces have at least one puncture other than the special one was imposed since these spaces were used to construct Hamiltonian

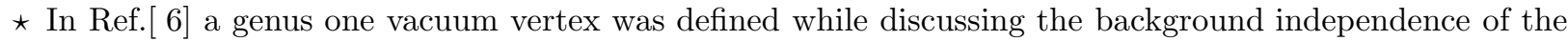
genus one contribution to the free energy. Still, there was no canonical choice for this vacuum vertex.
} 
functions, and field independent terms in the Hamiltonian drop out from background independence conditions. The subspaces are defined recursively as homotopies between the symmetric string vertices $\mathcal{V}$ and new spaces $\mathcal{V}^{\prime}$ (see Eqn. (5.1) of [7])

$$
\partial \mathcal{B} \simeq \mathcal{V}^{\prime}-\underline{\mathcal{V}}, \quad \text { with } \quad \mathcal{V}^{\prime}=\kappa \mathcal{V}_{0,3}^{\prime}+\kappa \mathcal{K} \mathcal{V}-\hbar \Delta \mathcal{B}-\{\mathcal{V}, \mathcal{B}\}
$$

The symbol $\simeq$ means that the equality holds up to the local coordinates at the special puncture, and up to surfaces of genus one with one special puncture and no ordinary puncture. Note that we have inserted the required factors of $\kappa$.

We need better control over the $\mathcal{B}$ spaces since we will insert dilatons on them, and dilatons capture some information about the local coordinates at the special puncture. We first claim that apart from surfaces having no ordinary punctures (3.10) can be made to hold strongly by careful definition of the way we insert the extra puncture. Thus we claim that in the sector where $n \geq 2$ (and $n \geq 3$ for $g=0$ ) we can find $\mathcal{B}$ spaces such that

$$
(\partial \mathcal{B})_{n \geq 2}=\left(\kappa \mathcal{V}_{0,3}^{\prime}+\kappa \overline{\mathcal{K}} \mathcal{V}-\hbar \Delta \mathcal{B}-\{\mathcal{V}, \mathcal{B}\}-\underline{\mathcal{V}}\right)_{n \geq 2} .
$$

Note that we have replaced the operator $\mathcal{K}$ which used no specific way of introducing coordinates at the extra puncture by $\overline{\mathcal{K}}$ which introduces coordinates using the minimal area metric on the surface. This equation can be established recursively. Note that we can proceed order by order in the dimensionality of the $\mathcal{B}$ spaces since the boundary of an $r$-dimensional $\mathcal{B}$ space on the left-hand side of the equation can only involve $\mathcal{B}$ spaces of dimensionality lower than $r$ on the right-hand side. The unique $\mathcal{B}$ space of lowest dimension is the one-dimensional space $\mathcal{B}_{0,3}$. To lowest order this equation requires $\partial \mathcal{B}_{0,3}=\mathcal{V}_{0,3}^{\prime}-\underline{\mathcal{V}}_{0,3}$. Since both $\mathcal{V}_{0,3}^{\prime}$ and $\mathcal{V}_{0,3}$ have well defined coordinates around the special puncture, we can clearly find an interpolating $\mathcal{B}_{0,3}$ with well-defined local coordinates around the special puncture. To guarantee we can solve (3.11) recursively we must establish that acting on the right-hand side of this equation with $\partial$ must give zero, since the right hand side is supposed to be the boundary of a space. Since $\mathcal{V}_{0,3}^{\prime}$ is just a point, we must show that

$$
(\kappa \partial \overline{\mathcal{K}} \mathcal{V}+\hbar \Delta \partial \mathcal{B}-\{\partial \mathcal{V}, \mathcal{B}\}+\{\mathcal{V}, \partial \mathcal{B}\}-\partial \underline{\mathcal{V}})_{n \geq 2}=0
$$

In order to find out if this equation holds we need to use the expression for $\partial \mathcal{B}$. We can actually use the expression in (3.11) since the terms in $\partial \mathcal{B}$ with no ordinary punctures, of which we 
do not keep track, cannot contribute to the left hand side of (3.12). This left hand side then becomes

$$
\begin{aligned}
{[-} & \kappa\{\overline{\mathcal{K}} \mathcal{V}, \mathcal{V}\}-\hbar \kappa \Delta \overline{\mathcal{K}} \mathcal{V}-\kappa\left\{\mathcal{V}_{0,3}^{\prime}, \mathcal{V}\right\} \\
& +\hbar\left(\kappa \Delta \mathcal{V}_{0,3}^{\prime}+\kappa \Delta \overline{\mathcal{K}} \mathcal{V}-\{\Delta \mathcal{V}, \mathcal{B}\}+\{\mathcal{V}, \Delta \mathcal{B}\}-\Delta \underline{\mathcal{V}}\right) \\
& +\frac{1}{2}\{\{\mathcal{V}, \mathcal{V}\}, \mathcal{B}\}+\hbar\{\Delta \mathcal{V}, \mathcal{B}\} \\
& +\left\{\mathcal{V}, \kappa \mathcal{V}_{0,3}^{\prime}+\kappa \overline{\mathcal{K}} \mathcal{V}-\hbar \Delta \mathcal{B}-\{\mathcal{V}, \mathcal{B}\}-\underline{\mathcal{V}}\right\} \\
& +\{\underline{\mathcal{V}}, \mathcal{V}\}+\hbar \Delta \underline{\mathcal{V}}]_{n \geq 2},
\end{aligned}
$$

where we made use of the strong equalities listed in (3.4), and for the help of the reader each term in (3.12) has been written as one line in (3.13). Note that the term $\hbar \kappa \Delta \mathcal{V}_{0,3}^{\prime}$ appearing in the second line, does not carry an ordinary puncture, hence it must be dropped. Using the exchange property of the antibracket (2.23), and the Jacobi identity (2.24), it is easily checked that all terms in (3.13) cancel out. This verifies the consistency condition. Thus the $\mathcal{B}$ spaces, with $n \geq 2$ can be defined recursively as homotopies between two spaces with coincident boundaries (see Ref. [6] for the construction of homotopies). This proves (3.11).

Since the $\mathcal{B}$ spaces do not contain surfaces with just one puncture, Eqn. (3.10) implies that

$$
\left(\mathcal{V}^{\prime}-\underline{\mathcal{V}}\right)_{g, 1} \approx 0 \quad \text { for } \quad g \geq 2
$$

Since all the $\mathcal{B}$ spaces were defined by the recursive construction above, Eqn. (3.14) must be verified to hold. This was done in Ref.[ 7], sec. 5.2. We do not think that equation (3.14) can be made to hold strongly by any choice of the $\mathcal{B}$ spaces. Thus we will use the failure of (3.14) to hold strongly to define $\mathcal{B}$ spaces with $n=1$, that is $\mathcal{B}$ spaces with no ordinary punctures. We attempt to set

$$
\partial \mathcal{B}_{g, 1} \equiv\left(\mathcal{V}^{\prime}-\underline{\mathcal{V}}\right)_{g, 1}=\overline{\mathcal{K}} \mathcal{V}_{g, 0}-\sum_{g_{1}=1}^{g-1}\left\{\mathcal{V}_{g_{1}, 1}, \mathcal{B}_{g-g_{1}, 2}\right\}-\Delta \mathcal{B}_{g-1,3}-\underline{\mathcal{V}}_{g, 1}, \quad(g \geq 2)
$$

The consistency of this definition requires that

$$
\partial\left(\mathcal{V}^{\prime}-\underline{\mathcal{V}}\right)_{g, 1}=0,
$$

and this equation can be verified to hold strongly with a calculation similar to the one we did 
in $(3.13)^{\star}$. It now follows that with our extended definition

$$
\mathcal{B} \equiv \sum_{g, n} \hbar^{g} \kappa^{2 g-2+n} \mathcal{B}_{g, n} \quad \text { with }\left\{\begin{array}{l}
n \geq 3 \text { for } g=0 \\
n \geq 2 \text { for } g=1 \\
n \geq 1 \text { for } g \geq 2
\end{array}\right.
$$

we have that

$$
\partial \mathcal{B}=\kappa \mathcal{V}_{0,3}^{\prime}+\kappa \overline{\mathcal{K}} \mathcal{V}-\hbar \Delta \mathcal{B}-\{\mathcal{V}, \mathcal{B}\}-\underline{\mathcal{V}}, \quad(g, n) \neq(1,1)
$$

Note that the consistency of this equation is guaranteed because the $n=1 \mathcal{B}$-spaces just introduced do not contribute to the right hand side $\left(\Delta \mathcal{B}_{g, 1}=0\right.$ and $\left.\left\{\mathcal{B}_{g, 1}, \mathcal{A}\right\}=0\right)$. The only piece of information that is not encoded in the above strong equation is given in (3.14) and now takes the form

$$
\partial \mathcal{B}_{g, 1} \approx 0, \quad(g \geq 2)
$$

We could try to eliminate the restriction to $(g, n) \neq(1,1)$ in $(3.18)$. The right hand side of (3.10) contains surfaces of genus one without an ordinary puncture. It is therefore tempting to introduce the space $\mathcal{B}_{1,1}$ such that $\partial \mathcal{B}_{1,1} \equiv\left(\mathcal{V}^{\prime}-\underline{\mathcal{V}}\right)_{1,1}=-\Delta \mathcal{B}_{0,3}-\underline{\mathcal{V}}_{1,1}$. This equation is not consistent since $\partial^{2} \mathcal{B}_{1,1} \neq 0$. This is not surprising, since in the analysis of [ 7$]$ the conditions of background independence of the free energy at genus one involved theory space connections, in addition to Riemann surfaces.

We can still fix (3.18) by hand, by subtracting from the right hand side the offending terms. We then have

$$
\partial \mathcal{B}=\kappa \mathcal{V}_{0,3}^{\prime}+\kappa \overline{\mathcal{K}} \mathcal{V}-\hbar \Delta\left(\mathcal{B}-\kappa \mathcal{B}_{0,3}\right)-\{\mathcal{V}, \mathcal{B}\}-\underline{\mathcal{V}}+\hbar \kappa \underline{\mathcal{V}}_{1,1}
$$

which is a strong equality without any qualifications. It is now useful to define

$$
\begin{aligned}
& \mathcal{B}_{>} \equiv \mathcal{B}-\kappa \mathcal{B}_{0,3} \\
& \mathcal{V}_{>} \equiv \mathcal{V}-\kappa \mathcal{V}_{0,3}
\end{aligned}
$$

and then rewrite the recursion relations as

$$
\partial \mathcal{B}_{>}=\kappa \overline{\mathcal{K}} \mathcal{V}-\hbar \Delta \mathcal{B}_{>}-\{\mathcal{V}, \mathcal{B}\}-\underline{\mathcal{V}}_{>}+\hbar \kappa \underline{\mathcal{V}}_{1,1}
$$

This is also a strong equality without qualifications.

\footnotetext{
$\star$ Since the verification just involves $\mathcal{B}$ spaces with $n \geq 2$, whose identities now hold strongly, the computation of [6], Eqn. (5.9) suffices.
} 


\section{The Dilaton Theorem}

In this section we begin by giving some motivation for the dilaton theorem. We explain what goes wrong when one tries to implement a dilaton shift as a gauge transformation generated by the state $|\chi\rangle$. This argument gives some insight into the kind of string field redefinition necessary to prove the dilaton theorem. We then turn to the precise statement of the dilaton theorem in string field theory, and explain what must be proven.

Motivation Consider an infinitesimal shift of the string field by the dilaton state $|D\rangle$. Since $|D\rangle=-Q|\chi\rangle$ this can be written as

$$
|\Psi\rangle \rightarrow|\Psi\rangle-\frac{\epsilon}{\kappa} Q|\chi\rangle
$$

This shift has the form of an infinitesimal gauge transformation, except that it is not a legal one, given that $b_{0}^{-}|\chi\rangle \neq 0$. Nevertheless it leaves the kinetic term of the action invariant, since

$$
\kappa \cdot \delta S_{0,2} \sim\left\langle\Psi\left|c_{0}^{-} Q Q\right| \chi\right\rangle+\left\langle D\left|c_{0}^{-} Q\right| \Psi\right\rangle=\left\langle D\left|c_{0}^{-} Q b_{0}^{-} c_{0}^{-}\right| \Psi\right\rangle=0
$$

The second equality follows by inserting the identity $1=\left\{b_{0}^{-}, c_{0}^{-}\right\}$, and the last equality follows by anticommuting $b_{0}^{-}$with $Q$ and using $\left(Q, b_{0}^{-}, L_{0}^{-}\right)|D\rangle=0$. Since (4.1) has succeeded to be a gauge transformation for the kinetic term of the closed string field theory, it is of interest to see if we can write a full nonlinear gauge transformation of the classical theory. The candidate gauge parameter is clearly $-\frac{1}{\kappa}|\chi\rangle$, and the naive gauge transformation would read [14]

$$
\begin{aligned}
\delta_{\chi}|\Psi\rangle & =\frac{\epsilon}{\kappa}|D\rangle+\frac{\epsilon}{\kappa} \sum_{n=3}^{\infty} \frac{\kappa^{n-2}}{(n-2) !}\left\langle V^{(n)} \mid \Psi\right\rangle^{n-2} \cdot|\chi\rangle|\mathcal{S}\rangle \\
& =\epsilon\left\{|\Psi\rangle, \frac{1}{\kappa} \mathbf{U}_{D(0,2)}+\sum_{n=3}^{\infty} \kappa^{n-3} f_{\chi}\left(\underline{\mathcal{V}}_{0, n}\right)\right\} .
\end{aligned}
$$

In the second step we use the results presented in section 2.5 to write the gauge transformation as a canonical transformation. The first nonlinear term corresponds to an insertion of the unrestricted state $|\chi\rangle$ on a three string vertex. Since $L_{0}^{-}|\chi\rangle=0$, and no antighost insertion is necessary, this term is independent of the choice of phase that is made at the special puncture. We have already seen that the leading variation of the action, of order $\mathcal{O}\left(\kappa^{-1}\right)$, vanishes. In addition, the $\mathcal{O}\left(\kappa^{0}\right)$ terms in the variation of the action also cancel,

$$
\left\{S_{0,2}, f_{\chi}\left(\underline{\mathcal{V}}_{0,3}\right)\right\}+\left\{f\left(\mathcal{V}_{0,3}\right), \mathbf{U}_{D(0,2)}\right\}=0
$$

The second equality follows from equations (2.41) and (2.44), since $\partial \mathcal{V}_{0,3}=0$. 
Since $|\chi\rangle$ is not in $\widehat{\mathcal{H}}$, in order to define the higher terms unambiguously, we must choose continuously over $\mathcal{V}_{0, n}$ a phase for the coordinate around the puncture where this state is inserted. In other words we must find a section in $\mathcal{P}_{0, n}$ over $\mathcal{V}_{0, n} \subset \widehat{\mathcal{P}}_{0, n}$. While this is not obviously feasible, it actually turns out that it can be done. Despite the existence of sections in $\mathcal{P}_{0, n}$ over $\mathcal{V}_{0, n}$, the $n \geq 4$ terms in (4.2) do not lead to an invariance of the action. The origin of the problem is the failure of the sections over $\mathcal{V}_{0, n}$ to satisfy the geometric recursion relations (3.8), which are crucial for proving gauge invariance of the classical action.

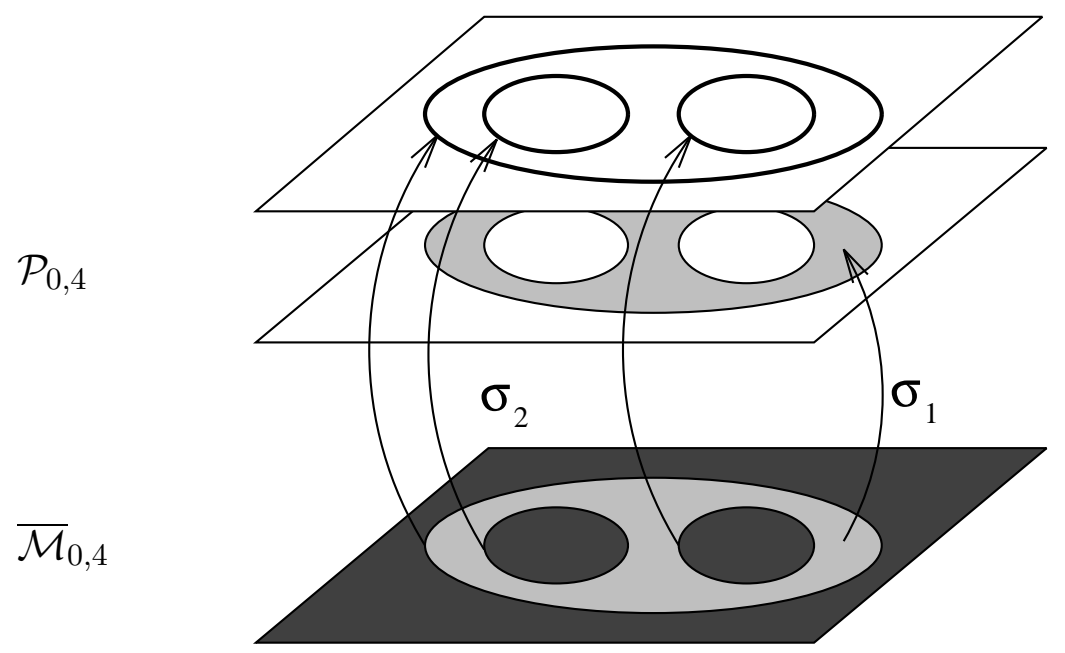

Figure 2. The lowest plane represents $\overline{\mathcal{M}}_{0,4}$, or equivalently its section in $\widehat{\mathcal{P}}_{0,4}$. The dark shaded regions represent the Feynman diagrams formed by sewing two threevertices $\mathcal{V}_{0,3}$, and the light shaded region is $\mathcal{V}_{0,4}$. The maps $\sigma_{1}$ and $\sigma_{2}$ give the sections in $\mathcal{P}_{0,4}$ over $\mathcal{V}_{0,4}$ and over the boundaries of the Feynman regions respectively. The section $\sigma_{1}$ cannot be deformed such that its boundary matches the section $\sigma_{2}$.

Let us consider the example of four-punctured spheres. Their compactified moduli space $\overline{\mathcal{M}}_{0,4}$ is itself a sphere. Three of the punctures are fixed, and the fourth is thought of as moving on this sphere. A local coordinate with a well-defined phase at the position of the fourth puncture defines a nonvanishing vector with a unique direction at that position; the direction corresponding to zero phase. As the puncture moves on the sphere it defines a vector

$\star$ One thinks of the punctures as moving in the plane, with three of them fixed at 0,1 and $\infty$. One can then take the phase of each puncture to be measured from the positive $x$-axis, for example. There is no global problem since the punctures never collide in $\mathcal{V}_{0, n}$. One cannot fix, however, the phase around a puncture in the higher genus $\mathcal{V}_{g, n}$ vertices, due to topological complications. There are no global sections in these cases. 
field, and the Poincaré-Hopf theorem requires such a vector field to have an index equal to two. In string field theory the space $\overline{\mathcal{M}}_{0,4}$ is decomposed into four regions: the region $\mathcal{V}_{0,4}$, and three disjoint regions corresponding to the Feynman diagrams formed by sewing two threevertices $\mathcal{V}_{0,3}$. The earlier choice of phases in $\mathcal{V}_{0,3}$ actually fixes the vector field in each of the Feynman regions. It turns out that in each of the Feynman regions the vector field has an index equal to +1 . Let us just assume that the index in each Feynman region is some integer $i_{F}$ (by symmetry it must be the same for the three regions). Since one must fix the phase continuously in $\mathcal{V}_{0,4}$, the vector field in this region will have index zero. Consequently, if the four vector fields defined over the four regions could be patched continuously to obtain a single vector field over the sphere, this resulting vector field would have index $3 i_{F}$. Since the vector field must have index two, this is impossible. Therefore the phase at the boundaries $-\frac{1}{2}\left\{\mathcal{V}_{0,3}, \mathcal{V}_{0,3}\right\}$ of the Feynman regions, cannot be made to agree with the phase at $\partial \mathcal{V}_{0,4}$, and the geometric recursion relation fails to hold in $\mathcal{P}_{0,4}$ (see Figure 2).

Since a dilaton shift cannot be extended to a true gauge transformation, we must look for a string field transformation that brings the action back to a recognizable form. That diffeomorphism of the string field will be seen to change the coupling constant of the theory. Since the first two terms of the pseudo-gauge transformation had the correct effect of preserving the action to order $\mathcal{O}\left(\kappa^{0}\right)$, we suspect those two terms will be present in the general diffeomorphism

$$
\delta|\Psi\rangle=\epsilon\left\{|\Psi\rangle, \frac{1}{\kappa} \mathbf{U}_{D(0,2)}+f_{\chi}\left(\underline{\mathcal{V}}_{0,3}\right)+\cdots\right\} .
$$

Precise Formulation In analogy with the proof of background independence [7], the dilaton theorem can be formulated precisely as an off-shell statement in string field theory. Consider two identical copies $\widehat{\mathcal{H}}_{\kappa}$ and $\widehat{\mathcal{H}}_{\kappa^{\prime}}$ of the state space $\widehat{\mathcal{H}}$. These copies are labeled by the value of the dimensionless coupling constant to be used in the construction of the corresponding string field theory. There should be a symplectic diffeomorphism

$$
F: \widehat{\mathcal{H}}_{\kappa^{\prime}} \rightarrow \widehat{\mathcal{H}}_{\kappa},
$$

that pulls back the relevant measure of a theory with coupling constant $\kappa$ to the measure of a theory with coupling constant $\kappa^{\prime}$, namely,

$$
F^{*}\left\{d \mu(\kappa) \exp \left(\frac{2}{\hbar} S(\kappa)\right)\right\}=d \mu\left(\kappa^{\prime}\right) \exp \left(\frac{2}{\hbar} S\left(\kappa^{\prime}\right)\right) .
$$

This means that an antibracket preserving field redefinition, which we expect to include a constant inhomogeneous term, maps a string theory with one coupling constant to a string theory with another coupling constant. It follows that the two string theories are just two different backgrounds of the same theory. 
For the case when the two coupling constants differ only infinitesimally,

$$
\kappa^{\prime}=(1+a \epsilon) \kappa
$$

with $a$ some constant to be fixed, an infinitesimal symplectic diffeomorphism must implement the desired map between the theories,

$$
F:|\Psi\rangle \rightarrow|\Psi\rangle+\epsilon\left\{|\Psi\rangle, \mathbf{U}_{D}\right\}
$$

where $\mathbf{U}_{D}$ is an odd Hamiltonian function. Our intuition about the dilaton tells us that $\mathbf{U}_{D}$ must begin by implementing a shift along the dilaton

$$
\mathbf{U}_{D}=\frac{1}{\kappa} \mathbf{U}_{D(0,2)}+\cdots, \quad \rightarrow \quad F:|\Psi\rangle \rightarrow|\Psi\rangle+\frac{\epsilon}{\kappa}|D\rangle+\cdots
$$

We must find the complete form of $\mathbf{U}_{D}$ and the value of the constant $a$. This will establish the infinitesimal form of the dilaton theorem.

It is interesting to consider the classical limit, where (4.6) requires $S\left(\kappa^{\prime}\right)=F^{*} S(\kappa)$, with $S$ now the classical master action (the limit of master action as $\hbar$ goes to zero). This means that

$$
S(\kappa+a \epsilon \kappa,|\Psi\rangle)=S\left(\kappa,|\Psi\rangle+\frac{\epsilon}{\kappa}|D\rangle+\cdots\right)
$$

This equation says that at the classical level a change of the string coupling constant can be achieved by giving the string field some expectation value in the direction of the dilaton (and performing a field redefinition).

Let us now derive the explicit form of the equation that $\mathbf{U}_{D}$ must satisfy. If we write $d \mu(\kappa)=\rho(\kappa) \prod d \psi$, one can show that [7]

$$
F^{*}(d \mu(\kappa))=\frac{\rho(\kappa)}{\rho\left(\kappa^{\prime}\right)} d \mu\left(\kappa^{\prime}\right)\left(1+2 \epsilon \Delta \mathbf{U}_{D}\right) \text {. }
$$

Moreover,

$$
F^{*}\{S(\kappa)\}=S(\kappa)+\epsilon\left\{S(\kappa), \mathbf{U}_{D}\right\}
$$

Equation (4.6) then reduces to

$$
a \kappa \frac{d}{d \kappa}\left(S+\frac{1}{2} \hbar \ln \rho\right)=\left\{S, \mathbf{U}_{D}\right\}+\hbar \Delta \mathbf{U}_{D} .
$$

This is the equation that must be satisfied by the odd Hamiltonian $\mathbf{U}_{D}$, for some value of the constant $a$. This equation is rather similar to the equation expressing the condition of 
background independence of the string field theory (Eqn. (4.16) of Ref.[ 7])

$$
\mathrm{D}_{\mu}(\widehat{\Gamma})\left(S+\frac{1}{2} \hbar \ln \rho\right)-\frac{1}{2} \hbar \operatorname{str} \widehat{\Gamma}_{\mu}=\left\{S, \mathbf{U}_{\mu}\right\}+\hbar \Delta \mathbf{U}_{\mu},
$$

where the aim was to find the Hamiltonian $\mathbf{U}_{\mu}$. Since the dilaton state is not marginal, the dilaton Hamiltonian $\mathbf{U}_{D}$ is expected to be somewhat more complicated than the background independence Hamiltonian, which could be expressed fully in terms of $\mathcal{B}$ spaces and the marginal operator as $\mathbf{U}_{\mu}=\mathbf{U}_{\mu(0,2)}-f_{\mu}(\mathcal{B})$. As discussed before Eqn. (4.4) we expect that

$$
\mathbf{U}_{D}=\frac{1}{\kappa} \mathbf{U}_{D(0,2)}+f_{\chi}\left(\underline{\mathcal{V}}_{0,3}\right)+\cdots,
$$

and finding the missing terms will be our objective. The full diffeomorphism will involve $\mathcal{B}$ and $\mathcal{V}$ spaces, and both $|D\rangle$ and $|\chi\rangle$. This will be derived in sect. 8. The correct value of $a$ turns out to be $a=+1$.

Using the form of the string action in (2.46) and the expression (3.7), the condition (4.13) for the dilaton theorem can be written more explicitly as

$$
\begin{aligned}
\left\{S, \mathbf{U}_{D}\right\}+\hbar \Delta \mathbf{U}_{D}= & a \hbar \kappa \frac{d}{d \kappa}\left(S_{1,0}+\frac{1}{2} \ln \rho\right) \\
& +a \sum_{g, n}(2 g-2+n) \hbar^{g} \kappa^{2 g-2+n} f\left(\mathcal{V}_{g, n}\right),
\end{aligned}
$$

where the sum over $g$ and $n$ runs precisely over the nonvanishing string vertices (Eqn. (3.7)). We have used the fact that the kinetic term $S_{0,2}$ is independent of $\kappa$. It is not clear to us whether the genus one terms included in the first line of the right hand side should be there. The naive expectation is that they should have no coupling constant dependence, but this expectation might not be realized. 


\section{Topology and Dilaton two-forms}

In this section we will begin by reviewing the Gauss-Bonnet theorem for the case of two dimensional Riemannian manifolds with boundaries. This theorem gives the Euler number in terms of an integral of curvature over the manifold and an integral of geodesic curvature over the boundaries. We will then calculate the general expressions for the dilaton two-form, and the $|\chi\rangle$ one-form when a generic family of local coordinates is used to produce the insertions. We then consider the case of a family of local normal-coordinates arising from a conformal metric on the surface. We show that the dilaton two-form becomes the curvature two form, a result familiar from Ref.[8], and the $|\chi\rangle$ one-form is seen to compute geodesic curvature. The latter result requires the definition of the phase of the local coordinate at the boundary of the surface. This can always be done in a canonical fashion.

\subsection{Gauss-Bonnet Theorem}

For a two dimensional Riemannian manifold $M$ with a smooth boundary $\partial M$, the GaussBonnet theorem states that the Euler number $\chi(M)$ of the manifold can be written as [17]

$$
\chi(M)=\frac{1}{2 \pi} \int_{M} R^{(2)}+\frac{1}{2 \pi} \int_{\partial M} k,
$$

where $R^{(2)}$ is the curvature 2 -form, and $k$ is geodesic curvature. For a genus $g$ surface $\Sigma$ with $n$ boundary components the Euler number is $\chi(\Sigma)=2-2 g-n$. Writing the area element as $d A=\rho^{2} d x \wedge d y=\frac{i}{2} \rho^{2} d z \wedge d \bar{z}$, the curvature 2-form is given by

$$
\begin{aligned}
R^{(2)} & =-\frac{4}{\rho^{2}} \partial \bar{\partial} \ln \rho d A \\
& =-2 i \partial \bar{\partial} \ln \rho(d z \wedge d \bar{z}) \\
& =i d(d z \partial \ln \rho-d \bar{z} \bar{\partial} \ln \rho) .
\end{aligned}
$$

It is of interest to give an explicit expression for the geodesic curvature $k$. The basic property of this curvature is that given an oriented open curve $\gamma$ with origin $x_{i}$ and endpoint $x_{f}$, embedded in a two dimensional manifold with a metric, the integral $\int_{\gamma} k$ of the geodesic curvature measures the rotation angle from the tangent $T\left(x_{i}\right)$ parallel transported to $x_{f}$ to the tangent $T\left(x_{f}\right)$ at $x_{f}$. When the curve is a geodesic we get zero, since geodesics are defined 
by parallel transporting the (unit) tangent vector. It is possible to find a local formula for the geodesic curvature $k$ in a region $R$ described by the uniformizer $z$. We find

$$
k=\mathrm{d} \theta_{\gamma}-i[d z \partial \ln \rho-d \bar{z} \bar{\partial} \ln \rho] .
$$

In this expression the term $i[\cdots]$ computes the rotation angle of any vector after parallel transport from $z$ to $z+d z$. The first term $\mathrm{d} \theta_{\gamma}$ computes the rotation angle of the tangent to the curve $\gamma$. The difference computes the angle from the parallel-transported tangent to the new tangent. Note that $k$ depends on the choice of curve $\gamma$. Consequently $k$ is a well-defined one-form on this curve, but not on the manifold $M$ in which it sits. At each point $p \in M$ one finds many geodesic curvature one-forms, depending on the chosen curve through that point. Acting with the exterior derivative on $k$ produces a two-form that is well-defined on $M$, and is given precisely by the curvature

$$
d k=-R^{(2)}
$$

When a two dimensional manifold $\Sigma \in \mathcal{V}_{g, n}$ is equipped with a minimal area metric, the integral of bulk curvature over the surface minus its unit disks $\left(\Sigma-\cup D_{i}\right)$ is given by

$$
\frac{1}{2 \pi} \int_{\Sigma-\cup D_{i}} R^{(2)}=\chi\left(\Sigma-\cup D_{i}\right)=2-2 g-n .
$$

This follows because the coordinate curves of a punctured surface equipped with a minimal area metric are geodesics of that metric (see sect. 3.1). Therefore, there is no geodesic curvature contribution to $(5.1)$.

\subsection{Dilaton TWO-FORM AND $|\chi\rangle$ ONE-FORM}

Consider once again the family of local coordinates (2.16) over a region $R$ of a Riemann surface, defined by

$$
z=h_{\lambda_{1} \lambda_{2}}(w)=z\left(\lambda_{1}, \lambda_{2}\right)+a\left(\lambda_{1}, \lambda_{2}\right) w+\frac{1}{2} b\left(\lambda_{1}, \lambda_{2}\right) w^{2}+\frac{1}{3 !} c\left(\lambda_{1}, \lambda_{2}\right) w^{3}+\cdots
$$

where $\lambda_{1}$ and $\lambda_{2}$ denote two real parameters parameterizing the domain $R$, which has a uniformizer $z$. We now define a dilaton two-form representing the measure for integrating a dilaton insertion

$$
\left|\omega_{D}^{(2)}\right\rangle \equiv d \lambda_{1} \wedge d \lambda_{2} \mathbf{b}\left(v_{\lambda_{1}}\right) \mathbf{b}\left(v_{\lambda_{2}}\right)|D\rangle
$$

Note that this is a two-form on the domain $R$, living in the state space of the conformal theory. The motivation for this definition is that $\left|\omega_{D}^{(2)}\right\rangle$ is clearly an ingredient in the construction of 
the canonical forms given in (2.27). It is now a short computation using (2.4), (2.32), and (2.33), to show that the two-form $\left|\omega_{D}^{(2)}\right\rangle$ only has a component along the vacuum state of the CFT,

$$
\left|\omega_{D}^{(2)}\right\rangle=|0\rangle \cdot \omega_{D}^{(2)}
$$

where the two-form $\omega_{D}^{(2)}$ on the region $R$ is given by

$$
\begin{aligned}
\omega_{D}^{(2)} & =d \lambda_{1} \wedge d \lambda_{2}\left[\left(\alpha_{\lambda_{2}} \gamma_{\lambda_{1}}-\alpha_{\lambda_{1}} \gamma_{\lambda_{2}}\right)-(\text { c.c. })\right] \\
& \left.=d \lambda_{1} \wedge d \lambda_{2}\left\{\left[\frac{d z}{d \lambda_{2}} \frac{d}{d \lambda_{1}}\left(\frac{b}{2 a^{2}}\right)-\frac{d z}{d \lambda_{1}} \frac{d}{d \lambda_{2}}\left(\frac{b}{2 a^{2}}\right)\right]-\text { (c.c. }\right)\right\}
\end{aligned}
$$

Since $\lambda_{1}$ and $\lambda_{2}$ parameterize the complex variable $z$ (the uniformizer on $R$ ), we can rewrite the above as

$$
\begin{aligned}
\omega_{D}^{(2)} & =-d z \wedge d\left(\frac{b}{2 a^{2}}\right)+d \bar{z} \wedge d\left(\overline{\frac{b}{2 a^{2}}}\right) \\
& =-d z \wedge d \bar{z}\left[\frac{\partial}{\partial \bar{z}}\left(\frac{b}{2 a^{2}}\right)+\frac{\partial}{\partial z}\left(\frac{\bar{b}}{2 \bar{a}^{2}}\right)\right]
\end{aligned}
$$

This is the desired result for the two-form representing a dilaton insertion. It is a completely general expression since it is constructed from the most general family of local coordinates.

For the $|\chi\rangle$ state it is natural to define a one form,

$$
\left|\omega_{\chi}^{(1)}\right\rangle \equiv \sum_{i=1,2} d \lambda_{i} \mathbf{b}\left(v_{\lambda_{i}}\right)|\chi\rangle
$$

Using (2.7), (2.32), and (2.33), we find

$$
\left|\omega_{\chi}^{(1)}\right\rangle=|0\rangle \cdot \omega_{\chi}^{(1)}
$$

where

$$
\begin{aligned}
\omega_{\chi}^{(1)} & =-\frac{1}{2} \sum_{i=1,2} d \lambda_{i}\left(\beta_{\lambda_{i}}-\bar{\beta}_{\lambda_{i}}\right) \\
& =\frac{1}{2} \mathrm{~d} \ln \left(\frac{a}{\bar{a}}\right)-d z\left(\frac{b}{2 a^{2}}\right)+d \bar{z}\left(\frac{\bar{b}}{2 \bar{a}^{2}}\right) .
\end{aligned}
$$

Note that the dilaton two form and the $|\chi\rangle$ one-form are related by

$$
\omega_{D}^{(2)}=-d \omega_{\chi}^{(1)}
$$

in agreement with (2.29), in view that $|D\rangle=-Q|\chi\rangle$. 
We saw in sect. 2.1 that the dilaton state $|D\rangle$ is contained in $\widehat{\mathcal{H}}$, but the state $|\chi\rangle$ is not. Moreover, as we stated in sect. 2.5, only states in $\widehat{\mathcal{H}}$ lead to insertions that are independent of the phase of the local coordinates. Therefore, the $|\chi\rangle$ insertion is expected to depend on the phase of the local coordinate, but the $|D\rangle$ insertion is not. We can now confirm this expectation. How does a change in phase alter the family of local coordinates (5.6)? Consider a fixed point $p_{0}$ corresponding to some fixed values of the parameters $\left(\lambda_{1}, \lambda_{2}\right)$, and the local coordinate $w$ around $p_{0}$. For any point $p$ near $p_{0}$ a change in the phase of the local coordinate amounts to $w(p) \rightarrow e^{-i \theta} w(p)$. The value $z(p)$ must remain unchanged when $w(p)$ changes, since the point $p$ does not move in the domain $R$, and therefore its coordinate on the $R$-uniformizer $z$ should not change. If we examine (5.6) we see that this requires $a \rightarrow e^{i \theta} a, b \rightarrow e^{2 i \theta} b$, etc. Under these transformations we see immediately that the dilaton form (5.10) is invariant, while the $|\chi\rangle$ one-form (5.13) transforms nontrivially, as expected.

When local coordinates arise from metrics. If the family of local coordinates we have been using to compute the dilaton two form happens to arise from a metric via the prescription reviewed in sect. 2.2, we can simplify and interpret our result. Using (2.21) in the expression for the two-form (5.10), we find

$$
\omega_{D}^{(2)}=2 d z \wedge d \bar{z} \partial \bar{\partial} \rho=i R^{(2)}(\rho)
$$

where, in the last step, we used the definition of curvature given in (5.2). For the state $|\chi\rangle$ we find

$$
\omega_{\chi}^{(1)}=i\left[\mathrm{~d} \theta_{a}-i(d z \partial \ln \rho-d \bar{z} \bar{\partial} \ln \rho)\right]
$$

where $\theta_{a}$ is the phase of $a$. We mentioned earlier that the prescription to find a family of local coordinates from a metric could not determine a phase for $a$. If we have a chosen curve $\gamma$ along which we will integrate the $|\chi\rangle$ insertion we can naturally fix $\theta_{a}=\theta_{\gamma}$, where $\theta_{\gamma}$ is the phase of the tangent to the curve $\gamma$ (all in the $z$-uniformizer). The tangent is defined uniquely by the orientation of the curve. With this identification we now find

$$
\omega_{\chi}^{(1)}=i k
$$

We have therefore shown that whenever local coordinates arise from metrics $|D\rangle$ insertions compute bulk curvature and $|\chi\rangle$ insertions compute geodesic curvature. 


\section{Main Integral Formulae}

The dilaton two-form $\left|\omega_{D}^{(2)}\right\rangle$ and $|\chi\rangle$ one-form $\left|\omega_{\chi}^{(1)}\right\rangle$ are used to integrate insertions of the corresponding states over fixed Riemann surfaces. This clearly does not suffice for our purposes. Since we deal with string amplitudes, the insertions must be further integrated over spaces of Riemann surfaces. We will see that for the $\mathcal{K}$-spaces and $\mathcal{L}$-spaces defined in sect. 3 , the insertion integrals over the surfaces and their boundaries can be isolated from the integrals over the rest of the data in these spaces (except possibly for the case of surfaces without punctures, which we will not address in this paper.). Such simplification was anticipated in Ref.[5], where it was observed that the dilaton state $|D\rangle$ is annihilated by the product of any three antighost oscillators of mode number greater than or equal to minus one. The novel points of our discussion are that all our expressions are well-defined since they never involve degenerate surfaces, we recognize the importance of including the $|\chi\rangle$ state in the integral statements, and the identities hold off-shell, namely other states appearing in the correlators need not be physical.

The state $|\chi\rangle$ can also be inserted into a space of surfaces by twist-sewing a three punctured sphere carrying $|\chi\rangle$ on a special puncture. We will discuss how the insertion of $|\chi\rangle$ depends on the choice of local coordinate at the special puncture. The results of the present section pave the way to the later construction of the diffeomorphism that establishes the dilaton theorem. They also illustrate how both $|D\rangle$ and $|\chi\rangle$ insertions must act simultaneously to produce results that only depend on Riemann surface data. This is important to understand CFT deformations.

\subsection{Dilaton and $|\chi\rangle$ Insertions on $\mathcal{K}$-Spaces and $\mathcal{L}$-Spaces}

Let $\mathcal{A}$ be a $d$-dimensional space of surfaces of genus $g$ with $n>1$ punctures $(n \geq 3$ for $g=0)$. We are interested in evaluating the integrals

$$
\begin{aligned}
f_{D}(\mathcal{K} \mathcal{A}) & \equiv \frac{1}{n !} \int_{\mathcal{K} \mathcal{A}}\left\langle\Omega^{[d+2] g, n+1} \mid \Psi\right\rangle_{1} \cdots|\Psi\rangle_{n}|D\rangle_{n+1} \\
f_{\chi}(\mathcal{L} \mathcal{A}) & \equiv \frac{1}{n !} \int_{\mathcal{L} \mathcal{A}}\left\langle\Omega^{[d+1] g, n+1} \mid \Psi\right\rangle_{1} \cdots|\Psi\rangle_{n}|\chi\rangle_{n+1}
\end{aligned}
$$

If $\mathcal{A}$ is a single surface, the results of the previous section for the dilaton two-form and the $|\chi\rangle$ one-form would suffice to evaluate the integrals. Recall that $\mathcal{K}$ and $\mathcal{L}$ insert states using some fixed but arbitrary family of local coordinates. 
We shall see that, for $\mathcal{K} \mathcal{A}$, the integration of the dilaton two-form over the surfaces can be carried out independently of the integration over the space $\mathcal{A}$. For $\mathcal{L} \mathcal{A}$, the integral of the $|\chi\rangle$ one-form over the boundaries of the surfaces can be carried out independently of the integration over the subspace $\mathcal{A}$. The integrals over the surfaces and boundaries depend in general on the surface, but as we shall see, their sum does not, and can therefore be factored out. In addition, for local coordinates arising from a minimal area metric on the surface the $|\chi\rangle$ integral vanishes, and the dilaton integral can be factored out alone.

Let $\left(\xi_{1}, \ldots, \xi_{d}\right)$ denote a set of coordinates for the space $\mathcal{A}$. It follows that $\mathcal{K} \mathcal{A}$ is then parameterized by $\left(\lambda_{1}, \lambda_{2}, \xi_{1}, \ldots, \xi_{d}\right)$, where $\lambda_{1}, \lambda_{2}$ are two real parameters associated to the position of the special puncture that $\mathcal{K}$ brings in. A family of local coordinates at the special puncture are defined by (5.6), where now the coefficients depend on all $d+2$ parameters. Namely, the local coordinate for the dilaton insertion depends on all the coordinates of the space $\mathcal{A}$, in addition to the position of the puncture. Since the surfaces have $n+1$ punctures, the Schiffer vectors must be $(n+1)$-tuples of vectors. For the case of the $|\chi\rangle$ insertion over $\mathcal{L} \mathcal{A}$ similar remarks apply, the only difference is that this space is $d+1$ dimensional.

Consider now the Schiffer vectors associated with the parameters $\lambda_{i}$. Since the $\mathcal{K}$-spaces and $\mathcal{L}$-spaces are defined by inserting an additional puncture on each surface $\Sigma \in \mathcal{A}$, these $(n+1)$-tuples cannot change any of the data on $\Sigma$. Therefore they need only be supported at the special puncture,

$$
\mathbf{v}_{\lambda_{i}}=\left(0, \ldots, 0, v_{\lambda_{i}}^{(n+1)}\right) \quad i=1,2
$$

where the last entry is the Schiffer vector computed in section 2.4, which moves the special puncture and changes its local coordinate.

Associated with the other $d$ parameters in the spaces $\mathcal{K} \mathcal{A}$ and $\mathcal{L} \mathcal{A}$, the Schiffer vectors can be written in the form

$$
\mathbf{v}_{\xi_{k}}=\widehat{\mathbf{v}}_{\xi_{k}}+\mathbf{v}_{\xi_{k}}^{\prime}, \quad k=1, \cdots, d
$$

where we have split the Schiffer vector into a vector $\widehat{\mathbf{v}}_{\xi_{k}}$, and a vector $\mathbf{v}_{\xi_{k}}^{\prime}$ of the form

$$
\widehat{\mathbf{v}}_{\xi_{k}}=\left(v_{\xi_{k}}^{(1)}, \ldots, v_{\xi_{k}}^{(n)}, 0\right), \quad \mathbf{v}_{\xi_{k}}^{\prime}=\left(0, \ldots, 0, v_{\xi_{k}}^{(n+1)}\right)
$$

The vector $\widehat{\mathbf{v}}_{\xi_{k}}$ is only supported on the original $n$ punctures of $\mathcal{A}$ and it changes the coordinate $\xi_{k}$ of $\mathcal{A}$. Since a change in this data must, in general, change the data at the special puncture, 
this Schiffer vector must have a component $\mathbf{v}_{\xi_{p}}^{\prime}$ on the special puncture. This component, however, need only change the local coordinate at the special puncture. It follows that

$$
\mathbf{b}\left(\mathbf{v}_{\xi_{p}}^{\prime}\right)|0\rangle_{n+1}=0
$$

since this antighost insertion only involves $b$-oscillators with mode number greater than or equal to zero.

Having understood the nature of the Schiffer variations, we can now use (2.27) to write the differential forms appearing in (6.1) as (suppressing the string field insertions)

$$
\begin{aligned}
\left\langle\Omega^{[d+2] g, n+1} \mid D\right\rangle_{n+1}= & (-2 \pi i)^{2-n-3 g} d \xi_{1} \wedge \cdots \wedge d \xi_{d} \wedge d \lambda_{1} \wedge d \lambda_{2} \\
& \cdot\left\langle\Sigma^{g, n+1}\left|\mathbf{b}\left(\mathbf{v}_{\xi_{1}}\right) \cdots \mathbf{b}\left(\mathbf{v}_{\xi_{d}}\right) \mathbf{b}\left(\mathbf{v}_{\lambda_{1}}\right) \mathbf{b}\left(\mathbf{v}_{\lambda_{2}}\right)\right| D\right\rangle_{n+1} \\
\left\langle\Omega^{[d+1] g, n+1} \mid \chi\right\rangle_{n+1}= & (-2 \pi i)^{2-n-3 g} d \xi_{1} \wedge \cdots \wedge d \xi_{d} \sum_{i=1,2} d \lambda_{i} \\
& \cdot\left\langle\Sigma^{g, n+1}\left|\mathbf{b}\left(\mathbf{v}_{\xi_{1}}\right) \cdots \mathbf{b}\left(\mathbf{v}_{\xi_{d}}\right) \mathbf{b}\left(\mathbf{v}_{\lambda_{i}}\right)\right| \chi\right\rangle_{n+1} .
\end{aligned}
$$

We now recognize that the dilaton two-form defined in (5.7) and the $|\chi\rangle$ one-form defined in (5.11) appear above. Using (5.8) and (5.12) we can write the above equations as

$$
\begin{aligned}
\left\langle\Omega^{[d+2] g, n+1} \mid D\right\rangle_{n+1} & =(-2 \pi i)^{2-n-3 g} d \xi_{1} \wedge \cdots \wedge d \xi_{d} \wedge \omega_{D}^{(2)}\left\langle\Sigma^{g, n+1}\left|\mathbf{b}\left(\mathbf{v}_{\xi_{1}}\right) \cdots \mathbf{b}\left(\mathbf{v}_{\xi_{d}}\right)\right| 0\right\rangle_{n+1} \\
\left\langle\Omega^{[d+1] g, n+1} \mid \chi\right\rangle_{n+1} & =(-2 \pi i)^{2-n-3 g} d \xi_{1} \wedge \cdots \wedge d \xi_{d} \wedge \omega_{\chi}^{(1)}\left\langle\Sigma^{g, n+1}\left|\mathbf{b}\left(\mathbf{v}_{\xi_{1}}\right) \cdot \mathbf{b}\left(\mathbf{v}_{\xi_{d}}\right)\right| 0\right\rangle_{n+1}
\end{aligned}
$$

Note that the state at the $(n+1)$-th puncture is the vacuum state. It cannot yet be brought all the way to the surface state $\langle\Sigma|$ due to the antighost insertions, that may have support on this puncture. Nevertheless, using (6.3) and (6.5), we see that

$$
\begin{aligned}
\left\langle\Sigma^{g, n+1}\left|\mathbf{b}\left(\mathbf{v}_{\xi_{1}}\right) \cdots \mathbf{b}\left(\mathbf{v}_{\xi_{d}}\right)\right| 0\right\rangle_{n+1} & =\left\langle\Sigma^{g, n+1}\left|\mathbf{b}\left(\widehat{\mathbf{v}}_{\xi_{1}}\right) \cdots \mathbf{b}\left(\widehat{\mathbf{v}}_{\xi_{d}}\right)\right| 0\right\rangle_{n+1} \\
& =\left\langle\Sigma^{g, n}\right| \mathbf{b}\left(\widehat{\mathbf{v}}_{\xi_{1}}\right) \cdots \mathbf{b}\left(\widehat{\mathbf{v}}_{\xi_{d}}\right)
\end{aligned}
$$

where in the last step the vacuum state can go freely all the way to the bra and delete the 
extra puncture. This enables us to rewrite the first of Eqns. (6.7) as

$$
\begin{aligned}
\left\langle\Omega^{[d+2] g, n+1} \mid D\right\rangle_{n+1} & =(-2 \pi i)^{2-n-3 g} d \xi_{1} \wedge \cdots \wedge d \xi_{d} \wedge \omega_{D}^{(2)} \cdot\left\langle\Sigma^{g, n}\right| \mathbf{b}\left(\widehat{\mathbf{v}}_{\xi_{1}}\right) \cdot \mathbf{b}\left(\widehat{\mathbf{v}}_{\xi_{d}}\right) \\
& =(-2 \pi i)^{-1}\left\langle\Omega^{[d] g, n}\right| \wedge \omega_{D}^{(2)}
\end{aligned}
$$

and similarly, the second one as

$$
\left\langle\Omega^{[d+1] g, n+1} \mid \chi\right\rangle_{n+1}=(-2 \pi i)^{-1}\left\langle\Omega^{[d] g, n}\right| \wedge \omega_{\chi}^{(1)} .
$$

The functionals of (6.1) then become

$$
\begin{aligned}
f_{D}(\mathcal{K} \mathcal{A}) & =\frac{1}{n !} \int_{\mathcal{A}}\left[\left\langle\Omega^{[d] g, n} \mid \Psi\right\rangle_{1} \cdots|\Psi\rangle_{n} \cdot \frac{i}{2 \pi} \int_{\Sigma-\cup D_{i}} \omega_{D}^{(2)}\right] \\
f_{\chi}(\mathcal{L A}) & =\frac{1}{n !} \int_{\mathcal{A}}\left[\left\langle\Omega^{[d] g, n} \mid \Psi\right\rangle_{1} \cdots|\Psi\rangle_{n} \cdot \frac{i}{2 \pi} \int_{\partial\left(\Sigma-\cup D_{i}\right)} \omega_{\chi}^{(1)}\right] .
\end{aligned}
$$

We have thus succeeded in isolating the two dimensional dilaton integral and the one-dimensional $|\chi\rangle$ integral from the integral over the coordinates of the space $\mathcal{A}$. If the local coordinates for the insertions come from a metric on the surfaces we can use (5.15) and (5.17) to write

$$
\begin{aligned}
f_{D}(\mathcal{K} \mathcal{A}) & =-\frac{1}{n !} \int_{\mathcal{A}}\left[\left\langle\Omega^{[d] g, n} \mid \Psi\right\rangle_{1} \cdots|\Psi\rangle_{n} \cdot \frac{1}{2 \pi} \int_{\Sigma-\cup D_{i}} R^{(2)}\right] \\
f_{\chi}(\mathcal{L} \mathcal{A}) & =-\frac{1}{n !} \int_{\mathcal{A}}\left[\left\langle\Omega^{[d] g, n} \mid \Psi\right\rangle_{1} \cdots|\Psi\rangle_{n} \cdot \frac{1}{2 \pi} \int_{\partial\left(\Sigma-\cup D_{i}\right)} k\right] .
\end{aligned}
$$

Each of these integrals depends on the metric that we use for the surfaces in $\mathcal{A}$. The sum, however, does not. Since the Euler number of the bordered surface $\Sigma-\cup D_{i}$ is $(2-2 g-n)$, the Gauss-Bonnet theorem (5.1) implies that

$$
f_{D}(\mathcal{K} \mathcal{A})+f_{\chi}(\mathcal{L A})=(2 g-2+n) f(\mathcal{A}) .
$$

This is an important result. It is independent of the metrics defined on the surfaces and used to derive the local coordinates. It is therefore well defined on spaces of Riemann surfaces. 
A particular case of the above general result will be useful to us. As we defined in sect. 3.1, a space of surfaces $\mathcal{V}_{i}$ is one in which the surfaces are equipped with unit disks that arise from minimal area metrics. In this case the coordinate curves are geodesics and $f_{\chi}\left(\mathcal{L} \mathcal{V}_{i}\right)$ vanishes. We then find that (6.13) gives

$$
f_{D}\left(\overline{\mathcal{K}} \mathcal{V}_{i}\right)=\left(2 g_{i}-2+n_{i}\right) f\left(\mathcal{V}_{i}\right)
$$

This result will be necessary in sect. 8 for the construction of the diffeomorphism establishing the dilaton theorem.

Using the $\mathcal{K}^{*}$ operator At the end of sect. 3.1 we mentioned the possibility of using a $\mathcal{K}^{*}$ operator that would insert a puncture with local coordinates induced from a metric which is a deformation of the minimal area metric on the interior of the surface minus its unit disks. The metric is left unchanged in some neighborhood of the coordinate curves. The purpose of this deformation is to produce metrics with smooth curvature. It is clear that for $\mathcal{K}^{*}$ insertions

$$
f_{D}\left(\mathcal{K}^{*} \mathcal{V}_{i}\right)=\left(2 g_{i}-2+n_{i}\right) f\left(\mathcal{V}_{i}\right)
$$

since, as was the case with $\overline{\mathcal{K}}$ there is no geodesic curvature contribution. The following identities hold

$$
\begin{aligned}
f_{D}\left(\mathcal{K}^{*}\left(\left\{\mathcal{V}_{1}, \mathcal{V}_{2}\right\}\right)-\left\{\mathcal{K}^{*} \mathcal{V}_{1}, \mathcal{V}_{2}\right\}-\left\{\mathcal{V}_{1}, \mathcal{K}^{*} \mathcal{V}_{2}\right\}\right) & =0, \\
f_{D}\left(\mathcal{K}^{*}\left(\Delta \mathcal{V}_{i}\right)-\Delta\left(\mathcal{K}^{*} \mathcal{V}_{i}\right)\right) & =0, \\
{\left[\partial, \mathcal{K}^{*}\right] \mathcal{V}_{i}+\left\{\mathcal{V}_{0,3}^{\prime}, \mathcal{V}_{i}\right\} } & =0 .
\end{aligned}
$$

The first two identities are verified using (6.15) and the first two equations in (2.41). These equations hold strongly even though the spaces of surfaces inside the big parentheses do not cancel out, as they do for the $\overline{\mathcal{K}}$ operator (see Eqn. (3.4)). The last identity holds because the metric is not changed in some neighborhood of the coordinate curves.

The reader may recall that the recursive construction of $\mathcal{B}$ spaces satisfying strong identities made use of $\overline{\mathcal{K}}$ and its properties. As we see, $\mathcal{K}^{*}$ satisfies similar properties, as long as dilatons are inserted on the special puncture. While we have not carried out the analysis in detail, we believe that a discussion similar to that in sect. 3.2 would yield a sequence of $\mathcal{B}$ spaces such that the recursion relations (3.20), with $\overline{\mathcal{K}}$ replaced by $\mathcal{K}^{*}$, would hold upon inserting a dilaton at the special puncture. This is all we would need to prove the dilaton theorem using $\mathcal{K}^{*}$, as it will be clear in sect. 8 . 


\subsection{Inserting $|\chi\rangle$ By TWist-SEWing}

The operation $\mathcal{L}$ acting on a space of surfaces $\mathcal{A}$ was defined to add a puncture over the boundary $\partial\left(\Sigma-\cup D_{i}\right)$ of every surface $\Sigma \in \mathcal{A}$. The local coordinate at the puncture is taken to arise from a metric on the surface. The phase of that local coordinate can be fixed using the tangent vector at the boundary, as explained at the end of sect. 5 . There is another way of inserting a puncture over the boundary $\partial\left(\Sigma-\cup D_{i}\right)$ of every surface $\Sigma \in \mathcal{A}$. Let $\mathcal{V}_{0,3}^{*}$ be a standard two-punctured sphere, with an additional special puncture. The local coordinates around the first and second punctures, located at $z=0$ and at $z=\infty$, are given by

$$
z_{1}=z, \quad z_{2}=1 / z
$$

where $z$ is a uniformizer. The local coordinate at the special puncture situated at $z=1$ is given by

$$
z_{3}=a(z-1)+b(z-1)^{2}+\cdots .
$$

The operation of twist-sewing $\mathcal{V}_{0,3}^{*}$ into a space of surfaces will have the effect of inserting a puncture precisely on $\partial\left(\Sigma-\cup D_{i}\right)$ for every surface $\Sigma$ in the space, as noticed in (3.6) for the special case of $\overline{\mathcal{L}}$ and the particular sphere $\mathcal{V}_{0,3}^{\prime}$. The local coordinate at the puncture will depend on the choice (6.18) made in defining $\mathcal{V}_{0,3}^{*}$. Note that the local coordinates on $\mathcal{V}_{0,3}^{*}$ are well-defined even including phases.

The objective in the present section is to evaluate $f_{\chi}\left(\left\{\mathcal{V}_{0,3}^{*}, \mathcal{A}\right\}^{\prime}\right)$ where $\mathcal{A}$ is some arbitrary space of surfaces. We use a prime on the antibracket to distinguish it from the standard antibracket defined for symmetric spaces. Since the space $\mathcal{V}_{0,3}^{*}$ is not symmetric, in $\left\{\mathcal{V}_{0,3}^{*}, \mathcal{A}\right\}^{\prime}$ we must choose one of the two ordinary punctures of $\mathcal{V}_{0,3}^{*}$ to do the sewing. We will choose the first puncture, and for the time being we will keep the choice of the local coordinate at the special puncture open. Later on we will consider the simplification that follows when the sphere $\mathcal{V}_{0,3}^{*}$ is required to be symmetric under the exchange of the ordinary punctures at zero and infinity.

We can begin our evaluation of $f_{\chi}\left(\left\{\mathcal{V}_{0,3}^{*}, \mathcal{A}\right\}^{\prime}\right)$ by making use of (2.41) and (2.35)

$$
\begin{aligned}
f_{\chi}\left(\left\{\mathcal{V}_{0,3}^{*}, \mathcal{A}\right\}^{\prime}\right) & =(-)^{\mathcal{A}+1}\left\{f(\mathcal{A}), f_{\chi}\left(\mathcal{V}_{0,3}^{*}\right)\right\}^{\prime} \\
& =\frac{(-)^{\mathcal{A}+1}}{(n-1) !} \int_{\mathcal{A}}\left\langle\Omega_{1^{\prime} \cdots n}^{[k] g, n} \mid \Psi\right\rangle^{n-1}\left\langle V_{123}^{*} \mid \Psi\right\rangle_{2}|\chi\rangle_{3}\left|\mathcal{S}_{11^{\prime}}\right\rangle \\
& =\frac{(-)^{\mathcal{A}}}{(n-1) !} \int_{\mathcal{A}}\left\langle\Omega^{[k] g, n} \mid \Psi\right\rangle^{n-1}\left\langle V_{123}^{*}\left|b_{0}^{-(1)}\right| \Psi\right\rangle_{2}|\chi\rangle_{3}\left|R_{11^{\prime}}\right\rangle
\end{aligned}
$$

To proceed further we need to evaluate $\left\langle V_{123}^{*}\right| b_{0}^{-(1)}$. To this end, we use the Ward identity 
$\left\langle V_{123}^{*}\right| \sum_{i} \oint_{\mathcal{C}_{i}} \frac{d z_{i}}{2 \pi i} b^{(i)}\left(z_{i}\right) v^{(i)}\left(z_{i}\right)=0$, where $v^{(i)}\left(z_{i}\right)$ is the restriction around the $i$-th puncture of a holomorphic vector field $v(z)$ on the sphere minus the punctures. An analogous relation holds for the antiholomorphic components. Taking $v(z)=z$, we find

$$
v^{(1)}=z_{1}, \quad v^{(2)}=-z_{2}, \quad v^{(3)}=a+\left(1+\frac{2 b}{a}\right) z_{3}+\mathcal{O}\left(z_{3}^{2}\right) .
$$

The Ward identity, together with its antiholomorphic counterpart, gives

$$
\left\langle V_{123}^{*}\right|\left[b_{0}^{-(1)}-b_{0}^{-(2)}+\left(a b_{-1}^{(3)}-\bar{a} \bar{b}_{-1}^{(3)}\right)+\left(1+\frac{b}{a}+\frac{\bar{b}}{\bar{a}}\right) b_{0}^{-(3)}+\left(\frac{b}{a}-\frac{\bar{b}}{\bar{a}}\right) b_{0}^{+(3)}+\cdots\right]=0,
$$

where the dots indicate terms of the form $b_{n}^{(3)}$ and $\bar{b}_{n}^{(3)}$ with $n \geq 1$. Since $b_{0}^{-}$annihilates the string field, and all antighost oscillators $b_{n \geq-1}, \bar{b}_{n \geq-1}$ except for $b_{0}^{-}$annihilate $|\chi\rangle$, we have that

$$
\left\langle V_{123}^{*}\left|b_{0}^{-(1)}\right| \Psi\right\rangle_{2}|\chi\rangle_{3}\left|R_{11^{\prime}}\right\rangle=\left(1+\frac{b}{a}+\frac{\bar{b}}{\bar{a}}\right)\left\langle V_{123}^{*} \mid 0\right\rangle_{3}|\Psi\rangle_{2}\left|R_{11^{\prime}}\right\rangle=\left(1+\frac{b}{a}+\frac{\bar{b}}{\bar{a}}\right)|\Psi\rangle_{1^{\prime}} .
$$

Back in (6.19) we then find

$$
f_{\chi}\left(\left\{\mathcal{V}_{0,3}^{*}, \mathcal{A}\right\}^{\prime}\right)=(-)^{\mathcal{A}} n\left(1+\frac{b}{a}+\frac{\bar{b}}{\bar{a}}\right) f(\mathcal{A}) .
$$

This is the equation we were after. It shows that line integration of a $|\chi\rangle$ insertion along the coordinate curves of surfaces amounts to multiplication by a constant determined by the geometry of a three string vertex that fixes the way $|\chi\rangle$ is inserted.

The three string vertex $\mathcal{V}_{0,3}^{*}$ we are going to use in our applications will be the vertex $\mathcal{V}_{0,3}^{\prime}$ discussed earlier, and fixed to be symmetric under the exchange of punctures one and two. That means that $z_{3}(z)=-z_{3}(1 / z)$. It is straightforward to show that this requirement on (6.18) leads to $b=-a / 2$. It then follows that for the symmetric vertex $\mathcal{V}_{0,3}^{\prime}$ the line integrals of $|\chi\rangle$ gives zero, namely $f_{\chi}\left(\left\{\mathcal{V}_{0,3}^{\prime}, \mathcal{A}\right\}^{\prime}\right)=0$. Since $\mathcal{V}_{0,3}^{\prime}$ is symmetric we can use the standard antibracket

$$
f_{\chi}\left(\left\{\mathcal{V}_{0,3}^{\prime}, \mathcal{A}\right\}\right)=0 .
$$

This result is intuitively plausible in the light that $|\chi\rangle$ insertions compute geodesic curvature. Assume a $|\chi\rangle$-insertion along the coordinate curve of some puncture is done via $\mathcal{L}$ using some metric on the surface. We expect that such an insertion can be reproduced by twist sewing with a fixed sphere $\mathcal{V}_{0,3}^{*}$ if the metric in some neighborhood of the coordinate curve is invariant under rigid rotations of the local coordinate at the puncture. If the coordinate curve is a geodesic of the metric we would expect that the sphere that reproduces the $\mathcal{L}$ insertion would have a symmetric coordinate at the special puncture. In this situation both the $\mathcal{L}$ insertion and the twist-sewing insertion would give zero. 


\section{CFT Deformations and String Backgrounds}

In this section we first use the dilaton to generate a CFT deformation. This deformation cannot be obtained in the standard way since the dilaton is not primary. It must involve the $|\chi\rangle$ state. We then show that this deformation is trivial. The second part is an attempt to give a sensible definition of a string background, and to argue that the dilaton deforms it in a nontrivial way.

\subsection{The CFT Deformation associated with the Dilaton}

The main question we want to address here is whether or not the dilaton state is an exactly marginal state. In other words we would like to know if this state generates an exact CFT deformation. When physicists discuss exactly marginal states of a matter conformal field theory they have in mind primary states $\left|\mathcal{O}_{M}\right\rangle$ of dimension $(1,1)$. When including the ghost CFT, one typically considers the dimension $(0,0)$ primary states $c_{1} \bar{c}_{1}\left|\mathcal{O}_{M}\right\rangle$. Since they are primary, these states lead to well defined two-forms on the surface, namely two-forms that are independent of the local coordinates used to insert the states. The insertions can therefore be integrated unambiguously over Riemann surfaces. This is essential since Riemann surfaces do not come equipped with canonical families of local coordinates. As we have seen in sect. 2.1, the dimension $(0,0)$ dilaton state $|D\rangle$ is not of the standard form and is not primary. As we will see, one cannot describe the deformation of a CFT just using $|D\rangle$, one must use the pair $(|D\rangle,|\chi\rangle)$ to be able to write something well defined on Riemann surfaces. After explaining how the deformation can be written, we will see that due to the ghost number anomaly it turns out to be trivial.

Now let us try to write explicitly a deformation induced by the dilaton. Given a surface state, a canonical deformation can be obtained by integration of the marginal operator over the surface minus its unit disks $[18,19,20]$

$$
\delta\langle\Sigma|=-\frac{\epsilon}{\pi} \int_{\Sigma-\cup D_{i}} d^{2} z\left\langle\Sigma ; z \mid \mathcal{O}_{M}\right\rangle .
$$

Writing this deformation in the language of forms we find

$$
\delta\left\langle\Omega_{\Sigma}^{[0] g, n}\right|=\epsilon \int_{\mathcal{K} \Sigma}\left\langle\Omega^{[2] g, n+1} \mid c \bar{c} \mathcal{O}_{M}\right\rangle_{(n+1)}
$$

where the zero-form on the left hand side is just the surface state up to a constant factor implicit 
in $(2.27)^{\star}$ Guided by the above equation, we attempt to define the dilaton deformation by

$$
\delta\left\langle\Omega_{\Sigma}^{[0] g, n}\right|=\epsilon \int_{\mathcal{K} \Sigma}\left\langle\Omega^{[2] g, n+1} \mid D\right\rangle_{(n+1)}
$$

The obvious difficulty with this expression is that since the dilaton is not primary, the integral is not well defined. If we put a metric on the surface and use it to obtain local normal-coordinates, the integral depends on the metric. Indeed, using (6.9) and (5.15) we see that

$$
\delta\left\langle\Omega_{\Sigma}^{[0] g, n}\right|=-\epsilon\left\langle\Omega^{[0] g, n}\right| \cdot \frac{1}{2 \pi} \int_{\Sigma-\cup D_{i}} R^{(2)} .
$$

Given that the integral depends on the chosen metric, this is not a well-defined deformation on a Riemann surface. The cure is clear from our earlier developments, we must include in the deformation a contribution from $|\chi\rangle$ that will give geodesic curvature.

$$
\delta\left\langle\Omega_{\Sigma}^{[0] g, n}\right|=\epsilon \int_{\mathcal{K} \Sigma}\left\langle\Omega^{[2] g, n+1} \mid D\right\rangle_{(n+1)}+\epsilon \int_{\partial(\mathcal{K} \Sigma)}\left\langle\Omega^{[1] g, n+1} \mid \chi\right\rangle_{(n+1)} .
$$

This will give

$$
\delta\left\langle\Omega_{\Sigma}^{[0] g, n}\right|=-\epsilon\left\langle\Omega^{[0] g, n}\right| \cdot\left(\frac{1}{2 \pi} \int_{\Sigma-\cup D_{i}} R^{(2)}+\frac{1}{2 \pi} \int_{\partial\left(\Sigma-\cup D_{i}\right)} k\right)
$$

and this time the sum of the integrals gives the Euler number for any choice of the conformal metric. Thus the above is well defined on Riemann surfaces. In terms of the surface states we then have

$$
\delta\left\langle\Sigma_{g, n}\right|=-\epsilon(2-2 g-n)\left\langle\Sigma_{g, n}\right|
$$

The deformation induced by the dilaton amounts to a scaling of the surface states of the conformal theory. Since the conformal theory is taken to have zero central charge, the operator formalism surface states have well defined normalization and it is possible to discuss the change of normalization.

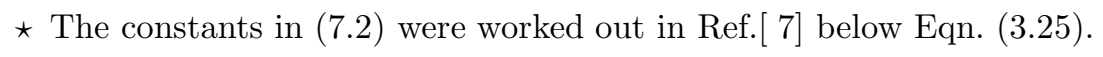


We can now verify explicitly that (7.7) is indeed a CFT deformation. In a CFT, surface states form a representation of the operation of sewing. Given two surfaces $\Sigma_{1}$ and $\Sigma_{2}$ which can be sewn together to form the surface $\Sigma_{1} \cup \Sigma_{2}$, the corresponding surface states must satisfy $\left\langle\Sigma_{1} \cup \Sigma_{2}\right|=\left\langle\Sigma_{1}\right|\left\langle\Sigma_{2} \mid R_{12}\right\rangle$, where the ket $\left|R_{12}\right\rangle$ is the inverse of the bra $\left\langle R_{12}\right|$ representing the canonical two-punctured sphere. It follows from (7.7) that $\delta\left\langle R_{12}\right|=0$, and therefore the inverse relation requires that $\delta\left|R_{12}\right\rangle=0$. We readily verify that we have a CFT deformation:

$$
\begin{aligned}
& \left(\left\langle\Sigma_{1}\right|+\delta\left\langle\Sigma_{1}\right|\right)\left(\left\langle\Sigma_{2}\left|+\delta\left\langle\Sigma_{2}\right|\right) \mid R_{12}\right\rangle\right. \\
& =\left\langle\Sigma_{1}\right|\left\langle\Sigma_{2} \mid R_{12}\right\rangle\left(1-\epsilon\left[2-2 g_{1}-n_{1}+2-2 g_{2}-n_{2}\right]\right), \\
& =\left\langle\Sigma_{1} \cup \Sigma_{2}\right|\left(1-\epsilon\left[2-2\left(g_{1}+g_{2}\right)-\left(n_{1}+n_{2}-2\right)\right]\right) \\
& =\left\langle\Sigma_{1} \cup \Sigma_{2}\right|+\delta\left\langle\Sigma_{1} \cup \Sigma_{2}\right| .
\end{aligned}
$$

Indeed we see that sewing the deformed states gives precisely the deformation of the sewn state. The question is whether the deformed CFT is new, or is just the old one in a different basis. To answer this question, consider the most general change of basis in the CFT

$$
|\Psi\rangle \rightarrow|\Psi\rangle-\epsilon A|\Psi\rangle
$$

where $A$ is an arbitrary but fixed matrix of constants in the CFT state space. Under this change of basis the surface states change as

$$
\left\langle\Sigma_{g, n}\right| \rightarrow\left\langle\Sigma_{g, n}\right|+\epsilon \sum_{i=1}^{n}\left\langle\Sigma_{g, n}\right| A^{(i)}
$$

so that the correlators are preserved. In here the superscript $i$ denotes the label of the state space that the matrix $A$ acts on. The transformation in (7.10) is a CFT deformation since, as one readily verifies, it preserves sewing. It is a trivial deformation because it arises from a change of basis. If the deformation in (7.7) is trivial it must follow that for some choice of $A$ one must have that

$$
\left\langle\Sigma_{g, n}\right|\left(2-2 g-n+\sum_{i=1}^{n} A^{(i)}\right)=0,
$$

for any choice of surface $\Sigma_{g, n}$. If we take $A=\mathbf{1}$, we see that we can eliminate the $n$ term out of the deformation. In fact, taking $A$ to be an arbitrary constant times the identity, we see that $\delta\left\langle\Sigma_{g, n}\right|=-\epsilon(2-2 g-\beta n)\left\langle\Sigma_{g, n}\right|$ is a CFT deformation for any value of the constant $\beta$. This shows that the $n$ term in (7.7) is completely ambiguous, and thus it is no surprise that Distler and Nelson found such ambiguities (see the discussion of Eqn. (1.1) in the introduction). 
This is not the end of the story, however. The term $(2-2 g)$ in the deformation is also trivial. This is due to the ghost number anomaly. Recall that the ghost current operator is not primary, and as a consequence the ghost number operator, ordinarily written as

$$
G=\sum_{n}: c_{n} b_{-n}+\bar{c}_{n} \bar{b}_{-n}:
$$

where the normal ordering is with respect to the $S L(2, C)$ vacuum, exhibits anomalous behavior. In particular, acting on surface states it gives

$$
\left\langle\Sigma_{g, n}\right| \sum_{i=1}^{n} G^{(i)}=(6-6 g)\left\langle\Sigma_{g, n}\right| .
$$

It now follows that (7.11) is satisfied by choosing $A=\mathbf{1}-G / 3$. This completes our proof that the dilaton deformation of a CFT is trivial.

\subsection{Dilatons Deform String Backgrounds}

There is more to a closed string background than a conformal field theory. In a conformal theory the natural objects are the surface states, which encode the information about the correlators on a fixed Riemann surface. The set of objects necessary to build a string field theory includes integrated correlators, or forms over moduli spaces of Riemann surfaces. While we could find a similarity transformation that canceled out the dilaton deformation of the surface states (the zero-forms), the same transformation cannot cancel out the dilaton deformation of the general forms. This is simple to see. Since the similarity operator was generated by ghost number, the antighost insertions necessary to build the general forms will change the counting that made the ghost number work for the zero-forms.

We will not try to propose a fully axiomatic definition of a string background. The following definition is just an attempt to capture the basic idea.

$\star$ In Ref.[21] the $\sigma$-model dilaton was coupled to the ghost current rather than to the curvature [22]. A better understanding of the relation between the two approaches might yield some insight into the fact that zero momentum dilatons do not deform the CFT.

$\dagger$ There have been some attempts to systematize the idea of a string background. See, for example [23], where the algebraic structure of classical string field theory was discussed, or the discussion based on homotopy Lie algebras and their extensions [ 14]. More recent works, by Kimura, Stasheff, and Voronov, $[24,25]$ also propose a definition of a (tree-level) string background that arises from a conformal theory. We believe that a physically motivated definition of a string background should not assume a conformal theory, and must incorporate loops. The definition we propose, in a somewhat different form, was suggested by E. Witten as a tool to attempt a definition of "theory space". 
String Background Let $\widehat{\mathcal{H}}$ be a Batalin-Vilkovisky vector space equipped with a function $S_{0,2}$, and let $\widehat{\mathcal{P}}$ denote the space of subspaces of $\cup_{g, n} \widehat{\mathcal{P}}_{g, n}$. A string background furnishes a map from $\widehat{\mathcal{P}}$ to the space of functions on $\widehat{\mathcal{H}}$. In other words, given a space of surfaces $\mathcal{A}_{g, n} \subset \widehat{\mathcal{P}}_{g, n}$ we are given a function $f\left(\mathcal{A}_{g, n}\right)$ in $\widehat{\mathcal{H}}$. This map must satisfy the consistency conditions

$$
\begin{aligned}
f(\Delta \mathcal{A}) & =-\Delta f(\mathcal{A}) \\
f(\{\mathcal{A}, \mathcal{B}\}) & =-\{f(\mathcal{A}), f(\mathcal{B})\} \\
f(\partial \mathcal{A}) & =-\left\{S_{0,2}, f(\mathcal{A})\right\} .
\end{aligned}
$$

This definition includes more than what we really need to construct a string field theory. A possible variant could be to demand the above conditions only for spaces $\mathcal{A}_{g, n}$ of the same dimension as $\mathcal{M}_{g, n}$. If we find an $f$ satisfying the above conditions, we have a string background. An obvious $f$ was given in (2.34).

We can now think of deformations. The obvious one to try is

$$
f\left(\mathcal{A}_{g, n}\right) \rightarrow f\left(\mathcal{A}_{g, n}\right)(1-\epsilon[2-2 g-n])
$$

which would follow by integrating, for each surface in the space, a dilaton over the surface minus its unit disks, and $|\chi\rangle$ over the boundaries of the disks. As we saw in sect. 6, the integral over the space $\mathcal{A}$ does not interfere with the integrals over the surfaces. In fact the above deformation is just the same as letting $f(\mathcal{A}) \rightarrow f(\mathcal{A})+\epsilon\left(f_{D}(\mathcal{K} \mathcal{A})+f_{\chi}(\mathcal{L} \mathcal{A})\right)$. In addition, we do not deform $S_{0,2}$. It is straightforward to verify that (7.15) is indeed a deformation of $f$ preserving Eqns. (7.14). It is therefore a deformation of a string background.

It follows from (2.34) that at the level of forms, the deformation postulated in (7.15) arises from the deformation

$$
\left\langle\Omega^{[d] g, n}\right| \rightarrow\left\langle\Omega^{[d] g, n}\right|(1-\epsilon[2-2 g-n])
$$

where $d$, the degree of the form, is equal to the number of antighost insertions. Acting on these forms the ghost number operator gives

$$
\left\langle\Omega^{[d] g, n}\right| \sum_{i=1}^{n} G^{(i)}=\left\langle\Omega^{[d] g, n}\right|(6-6 g+d),
$$

where use was made of (7.13) after commuting the ghost number operators through the antighost insertions (recall $[G, b]=-b$ ). It is clear from (7.16) and (7.17) that a similarity transformation induced by the ghost number operator will not be able to reproduce the 
deformation of the various forms. ${ }^{\ddagger}$ We believe there is no similarity transformation that can reproduce this deformation, namely we believe that the dilaton truly deforms a string background. The existence of a similarity transformation generating this deformation would imply that there is a homogeneous string field transformation that changes the coupling constant of string theory. This would make no sense, since it would mean that the string coupling constant is not an observable. The fact that a shift of the string field is necessary (along with a linear and higher order transformations) to change the string field coupling constant, is essential to have a change in the physics of the theory. Indeed, at the level of low energy Lagrangian, it is well known we must shift the dilaton to change the string field coupling constant.

\section{The Diffeomorphism Establishing the Dilaton Theorem}

In sect. 4 we gave the precise statement of the dilaton theorem. We concluded that we had to find a Hamiltonian function $\mathbf{U}_{D}$ satisfying (4.16)

$$
\begin{aligned}
\left\{S, \mathbf{U}_{D}\right\}+\hbar \Delta \mathbf{U}_{D}= & a \hbar \kappa \frac{d}{d \kappa}\left(S_{1,0}+\frac{1}{2} \ln \rho\right) \\
& +a \sum_{g, n}(2 g-2+n) \hbar^{g} \kappa^{2 g-2+n} f\left(\mathcal{V}_{g, n}\right) .
\end{aligned}
$$

On the right hand side of this equation we included a term related to the one-loop free energy. Since the genus one contribution to the free energy $F_{g=1}$ is usually thought to be coupling constant independent, this term is a surprise. We will see that it may be necessary. We also saw in sect. 4 that the Hamiltonian function $\mathbf{U}_{D}$ is expected to be of the form (Eqn. (4.4))

$$
\mathbf{U}_{D}=\frac{1}{\kappa} \mathbf{U}_{D(0,2)}+f_{\chi}\left(\underline{\mathcal{V}}_{0,3}\right)+\cdots
$$

The object of the present section is to give the missing terms in the above equation and thus find the diffeomorphism that establishes the dilaton theorem. We claim that the complete diffeomorphism is given by

$$
\mathbf{U}_{D}=\frac{1}{\kappa}\left[\mathbf{U}_{D(0,2)}+f_{\chi}\left(\kappa \underline{\mathcal{V}}_{0,3}\right)-f_{D}\left(\mathcal{B}_{>}\right)+f_{\chi}\left(\left\{\kappa \mathcal{B}_{0,3}, \mathcal{V}\right\}\right)\right]
$$

where the subscript $>$ denotes that the $(0,3)$ space is not included (see Eqn. (3.21)). The third term is the same term that appears in the diffeomorphism implementing background

$\ddagger$ More precisely, the similarity transformation must be a canonical transformation, that is, it should preserve the symplectic structure in $\widehat{\mathcal{H}}$. The ghost number generated canonical transformation reads $\delta|\Psi\rangle=\epsilon(G-5 / 2)|\Psi\rangle$. Note that the similarity transformation $\delta|\Psi\rangle=\epsilon|\Psi\rangle$ is not canonical. When doing string field theory this redefinition changes the observables of the theory unless the symplectic form is changed accordingly (see sect. 6.1 .3 of Ref.[ 7]). 
independence except for the exclusion of $\mathcal{B}_{0,3}$. The last term is new, and was found by trial and error. Here the $|\chi\rangle$ state is inserted on the special puncture of the three-punctured spheres defining the space $\mathcal{B}_{0,3}$. Even though $|\chi\rangle$ is not in $\widehat{\mathcal{H}}$, this term is well-defined since we can define unambiguously a local coordinate with a phase on the special puncture of the spheres defining $\mathcal{B}_{0,3}$. It should be noted that the last two terms in (8.3) give contributions to the Hamiltonian that begin as cubic terms in the string field. They complete the quadratic Hamiltonian that was anticipated by the analysis of gauge transformations.

We now verify that the Hamiltonian $\mathbf{U}_{D}$ does indeed satisfy (8.1). The first term on the left hand side of (8.1) is given by

$$
\begin{aligned}
\left\{S, \mathbf{U}_{D}\right\}= & \frac{1}{\kappa}\left\{S, \mathbf{U}_{D(0,2)}\right\} \\
& +\left\{S, f_{\chi}\left(\underline{\mathcal{V}}_{0,3}\right)\right\} \\
& -\frac{1}{\kappa}\left\{S, f_{D}\left(\mathcal{B}_{>}\right)\right\} \\
& +\left\{S, f_{\chi}\left(\left\{\mathcal{B}_{0,3}, \mathcal{V}\right\}\right)\right\} .
\end{aligned}
$$

Using (2.47) and (2.48), we immediately find

$$
\begin{aligned}
\left\{S, \mathbf{U}_{D}\right\} & =\frac{1}{\kappa} f_{D}(\underline{\mathcal{V}}) \\
& -f_{D}\left(\underline{\mathcal{V}}_{0,3}\right)-f_{\chi}\left(\left\{\mathcal{V}, \underline{\mathcal{V}}_{0,3}\right\}\right) \\
& +\frac{1}{\kappa} f_{D}\left(\partial \mathcal{B}_{>}+\left\{\mathcal{V}, \mathcal{B}_{>}\right\}\right) \\
& -f_{D}\left(\left\{\mathcal{B}_{0,3}, \mathcal{V}\right\}\right)-f_{\chi}\left(\partial\left\{\mathcal{B}_{0,3}, \mathcal{V}\right\}+\left\{\mathcal{V},\left\{\mathcal{B}_{0,3}, \mathcal{V}\right\}\right\}\right),
\end{aligned}
$$

where each row in (8.5) is equal to the corresponding row in (8.4) . Collecting terms and using the Jacobi identity (2.24) in the fourth row gives

$$
\begin{aligned}
\left\{S, \mathbf{U}_{D}\right\} & =\frac{1}{\kappa} f_{D}\left(\partial \mathcal{B}_{>}+\{\mathcal{V}, \mathcal{B}\}+\underline{\mathcal{V}}_{>}\right) \\
& -f_{\chi}\left(\left\{\underline{\mathcal{V}}_{0,3}+\partial \mathcal{B}_{0,3}, \mathcal{V}\right\}+\left\{\mathcal{B}_{0,3}, \partial \mathcal{V}+\frac{1}{2}\{\mathcal{V}, \mathcal{V}\}\right\}\right)
\end{aligned}
$$

Using the geometrical recursion relations (3.8), the relation $\partial \mathcal{B}_{0,3}=\mathcal{V}_{0,3}^{\prime}-\underline{\mathcal{V}}_{0,3}$, and the vanishing of $f_{\chi}\left(\left\{\mathcal{V}_{0,3}^{\prime}, \mathcal{V}\right\}\right)$ (Eqn. (6.24)), we obtain

$$
\left\{S, \mathbf{U}_{D}\right\}=\frac{1}{\kappa} f_{D}\left(\partial \mathcal{B}_{>}+\{\mathcal{V}, \mathcal{B}\}+\underline{\mathcal{V}}_{>}\right)+f_{\chi}\left(\left\{\mathcal{B}_{0,3}, \hbar \Delta \mathcal{V}\right\}\right)
$$

This completes the calculation of the first term on the left hand side of (8.1). The second term 
is easily calculated using the first equation in (2.41), and the second equation in (2.26)

$$
\hbar \Delta \mathbf{U}_{D}=-\hbar f_{\chi}\left(\underline{\mathcal{V}}_{0,3}\right)+\frac{1}{\kappa} f_{D}\left(\hbar \Delta \mathcal{B}_{>}\right)-f_{\chi}\left(\left\{\mathcal{B}_{0,3}, \hbar \Delta \mathcal{V}\right\}\right)
$$

The last two equations add up to give

$$
\hbar \Delta \mathbf{U}_{D}+\left\{S, \mathbf{U}_{D}\right\}=\frac{1}{\kappa} f_{D}\left(\partial \mathcal{B}_{>}+\{\mathcal{V}, \mathcal{B}\}+\hbar \Delta \mathcal{B}_{>}+\underline{\mathcal{V}}_{>}\right)-f_{\chi}\left(\hbar \underline{\mathcal{V}}_{0,3}\right)
$$

Using the recursion relation (3.22) for the $\mathcal{B}$ spaces we finally find

$$
\hbar \Delta \mathbf{U}_{D}+\left\{S, \mathbf{U}_{D}\right\}=f_{D}(\overline{\mathcal{K}} \mathcal{V})+\hbar\left[f_{D}\left(\underline{\mathcal{V}}_{1,1}\right)-f_{\chi}\left(\Delta \underline{\mathcal{V}}_{0,3}\right)\right]
$$

We are now ready to verify (8.1). In order to do this, the first term in the right hand side is separated into terms having to do with ordinary string vertices, and terms having to do with vacuum vertices (for $g \geq 2$ )

$$
f_{D}(\overline{\mathcal{K}} \mathcal{V})=\sum_{g, n \geq 1} \hbar^{g} \kappa^{2 g-2+n} f_{D}\left(\overline{\mathcal{K}} \mathcal{V}_{g, n}\right)+\sum_{g \geq 2} \hbar^{g} \kappa^{2 g-2} f_{D}\left(\overline{\mathcal{K}} \mathcal{V}_{g, 0}\right)
$$

Using (6.14) for the $n \geq 1$ terms, we find

$$
f_{D}(\overline{\mathcal{K}} \mathcal{V})=\sum_{g, n \geq 1} \hbar^{g} \kappa^{2 g-2+n}(2 g-2+n) f\left(\mathcal{V}_{g, n}\right)+\sum_{g \geq 2} \hbar^{g} \kappa^{2 g-2} f_{D}\left(\overline{\mathcal{K}} \mathcal{V}_{g, 0}\right)
$$

Comparing with (8.1), we see that all the string field dependent terms of the equation can now be made to match by choosing $a=+1$. This shows that shifting the dilaton amounts to a shift in the coupling constant in all the string field dependent terms of the string action. This establishes the dilaton theorem as far as all off-shell amplitudes is concerned.

Comparing the string field independent terms in (8.1) we are led to the requirements

$$
f_{D}\left(\underline{\mathcal{V}}_{1,1}\right)-f_{\chi}\left(\underline{\mathcal{V}}_{0,3}\right)=\kappa \frac{d}{d \kappa}\left(S_{1,0}+\frac{1}{2} \ln \rho\right)
$$

at genus one, and

$$
f_{D}\left(\overline{\mathcal{K}} \mathcal{V}_{g, 0}\right)=(2 g-2) f\left(\mathcal{V}_{g, 0}\right)
$$

for $g \geq 2$. For the latter equation, (6.14) is not directly applicable since the surfaces in the moduli space $\mathcal{V}_{g, 0}$ have no punctures. One might compute $f_{D}\left(\overline{\mathcal{K}} \mathcal{V}_{g, 0}\right)$ by adding an auxiliary 
puncture, and then following the procedure of sect. 3.2. The genus one case is also unclear. Since $|D\rangle=-Q|\chi\rangle$, one might be tempted to use (2.42) to claim that

$$
f_{D}\left(\underline{\mathcal{V}}_{1,1}\right)=-f_{\chi}\left(\partial \underline{\mathcal{V}}_{1,1}\right)
$$

and the recursion relation

$$
\partial \mathcal{V}_{1,1}=-\hbar \Delta \mathcal{V}_{0,3}
$$

to conclude that the left hand side of (8.13) vanishes. However each step in this analysis is problematic. (8.15) only holds if there exists a section in $\mathcal{P}_{1,1}$ over $\mathcal{V}_{1,1}$, and it is not clear that

one does exist. In addition, (8.16) is only known to hold in $\widehat{\mathcal{P}}_{1,1}$ and may not hold in $\mathcal{P}_{1,1}$. We leave open the issue of vacuum graphs and the dilaton.

Acknowledgements: We wish to thank K. Ranganathan, A. Sen, and H. Sonoda for their constructive comments during the course of this research. One of us (O.B.) wishes to thank the Center for Theoretical Physics and the Department of Physics at M.I.T. where part of this work was done.

\section{REFERENCES}

1. H. Hata, 'Soft dilaton theorem in string field theory', Prog. Theor. Phys. 88(1992)1197.

2. T. Yoneya, 'String-coupling constant and dilaton vacuum expectation value in string field theory', Phys. Lett. B 197 (1987) 76.

3. H. Hata and Y. Nagoshi, 'Dilaton and classical solutions in pregeometrical string field theory', Prog. Th. Phys. 80 (1988) 1088.

4. T. Kugo and B. Zwiebach, 'Target space duality as a symmetry of string field theory', Prog. Th. Phys. 87 (1992) 801.

5. J. Distler and P. Nelson, 'Topological couplings and contact terms in 2-D field theory', Comm. Math. Phys. 138 (1991) 273; 'The Dilaton Equation in Semirigid String Theory', Nucl. Phys. B366 (1991) 255.

6. A. Sen and B. Zwiebach, 'Local background independence of classical closed string field theory', Nucl. Phys. B414 (1994) 649, hep-th/9307088.

7. A. Sen and B. Zwiebach, 'Quantum background independence of closed string field theory', Nucl. Phys. B423 (1994) 580, hep-th/9311009.

8. J. Polchinski, 'Factorization of bosonic string amplitudes', Nucl. Phys. B307 (1988) 61. 
9. P. B. Gilkey, Invariance Theory, the Heat Equation, and the Atiyah-Singer Index Theorem, Wilmington, Delaware, Publish or Perish, 1984.

10. A. Belopolsky and B. Zwiebach, 'Off-shell string amplitudes: Towards a computation of the tachyon potential', MIT preprint, MIT-CTP-2336, submitted to Nucl. Phys. B, hep-th/9409015.

11. P. Nelson, 'Covariant insertion of general vertex operators', Phys. Rev. Lett. 62(1989)993; H. S. La and P. Nelson, 'Effective field equations for fermionic strings', Nucl. Phys. B332 (1990) 83.

12. C. M. Becchi, R. Collina, and C. Imbimbo, 'On the semirelative condition for closed topological strings', Phys. Lett. B 322 (1994) 79.

13. E. Wong, 'Recursion Relations in Semirigid Topological Gravity', Int. Jour. Mod. Phys. A7 (1992) 6773.

14. B. Zwiebach, 'Closed string field theory: Quantum action and the Batalin-Vilkovisky master equation', Nucl. Phys B390 (1993) 33, hep-th/9206084.

15. A. Sen and B. Zwiebach, 'Background independent algebraic structures in closed string field theory', MIT-CTP-2346, August 1994, hep-th/9408053.

16. H. Sonoda and B. Zwiebach, 'Closed string field theory loops with symmetric factorizable quadratic differentials', Nucl. Phys. B331 (1990) 592.

17. I. M. Singer, and J. A. Thorpe, Lecture notes on elementary topology and geometry, New York: Springer Verlag (1976).

18. M. Campbell, P. Nelson and E. Wong, Int. Jour. Mod. Phys. A6 (1991) 4909.

19. K. Ranganathan, 'Nearby CFT's in the operator formalism: the role of a connection', Nucl. Phys. B408 (1993) 180.

20. K. Ranganathan, H. Sonoda and B. Zwiebach, 'Connections on the state-space over conformal field theories', Nucl. Phys. B414 (1994) 405, hep-th/9304053.

21. T. Banks, D. Nemeschansky and A. Sen, 'Dilaton coupling and BRST quantization of bosonic strings, Nucl. Phys B277 (1986) 67.

22. E. Fradkin and A. Tseytlin, Phys. Lett. 158B (1985) 316; 160B (1985) 64.

23. A. Sen, 'Some applications of string field theory', Proceedings of the Conference Strings and Symmetries, Stony Brook, May, 1991, World Scientific, p.355., hep-th/9109022.

24. T. Kimura, J. Stasheff, and A. A. Voronov, 'On operad structures of moduli spaces and string theory', Kyoto University preprint RIMS-936, July 1993, to appear in Comm. Math. Phys., hep-th/9307114. 
25. T. Kimura and A. A. Voronov, 'The cohomology of algebras over moduli spaces', University of North Carolina preprint, October 1994, hep-th/9410108. 